\title{
Zone of influence for particle number concentrations at signalised traffic intersections
}

\author{
Anju Goela, Prashant Kumara, b, * \\ aDepartment of Civil and Environmental Engineering, Faculty of Engineering and Physical \\ Sciences, University of Surrey, Guildford GU2 7XH, United Kingdom \\ bEnvironmental Flow (EnFlo) Research Centre, Faculty of Engineering and Physical \\ Sciences, University of Surrey, Guildford GU2 7XH, United Kingdom
}

\begin{abstract}
Estimation of zone of influences (ZoI) at signalised traffic intersections (TI) is important to accurately model particle number concentrations (PNCs) and their exposure to public at emission hotspot locations. However, estimates of ZoI for PNCs at different types of TIs are barely known. We carried out mobile measurements inside the car cabin with windows fully open for size-resolved PNCs in the 5-560 $\mathrm{nm}$ range on a $6 \mathrm{~km}$ long busy round route that had 10 TIs. These included four-way TIs without built-up area $\left(\mathrm{TI}_{4 \mathrm{w}}\right.$ $\mathrm{nb}$ ), four-way TIs with built-up area $\left(\mathrm{TI}_{4 \mathrm{w}-\mathrm{wb}}\right)$, three-way TIs without built-up area $\left(\mathrm{TI}_{3 \mathrm{w}-\mathrm{nb}}\right)$ and three-way TIs with built-up area $\left(\mathrm{TI}_{3 \mathrm{w}-\mathrm{wb}}\right)$. Mobile measurements were made with a fast response differential mobility spectrometer (DMS50). Driving speed and position of the car were recorded every second using a global positioning system (GPS). Positive matrix factorisation (PMF) modelling was applied on the data to quantify the contribution of PNCs released during deceleration, creep-idling, acceleration and cruising to total PNCs at the TIs. The objectives were to address the following questions: (i) how does ZoI vary at different types of TIs in stop- and go-driving conditions?, (ii)
\end{abstract}

${ }^{*}$ Corresponding author: Department of Civil and Environmental Engineering, Faculty of Engineering and Physical Sciences, University of Surrey, Guildford GU2 7XH, United Kingdom; Tel.: +44 1483 682762; Fax: +44 1483 682135; E-mail addresses: P.Kumar@surrey.ac.uk,Prashant.Kumar@cantab.net

Cite this article as: Goel, A., Kumar, P., 2015. Zone of influence for particle number concentrations at signalised traffic intersections, Atmospheric Environment 123, 25-38. Online Link: http://dx.doi.org/10.1016/j.atmosenv.2015.10.054 
what is the effect of different driving conditions on ZoI of a TI?, (iii) how realistically can the PNC profiles be generalised within a ZoI of a TI?, and (iv) what is the share of emissions during different driving conditions towards the total PNCs at a TI? Average length of ZoI in longitudinal direction and along the road was found to be highest (148 $\mathrm{m} ; 89$ to $-59 \mathrm{~m}$ from the centre of a TI) at a $\mathrm{TI}_{3 \mathrm{w}-\mathrm{wb}}$, followed by $\mathrm{TI}_{4 \mathrm{w}-\mathrm{nb}}(129 \mathrm{~m} ; 79$ to $42 \mathrm{~m}), \mathrm{TI}_{3 \mathrm{w}-\mathrm{nb}}(86 \mathrm{~m} ; 71$ to $-15 \mathrm{~m})$ and $\mathrm{TI}_{4 \mathrm{w}-\mathrm{wb}}(79 \mathrm{~m} ; 46$ to $-33 \mathrm{~m})$ in stop- and godriving conditions. During multiple stopping driving conditions when a vehicle stops at a TI more than once in a signal cycle due to oversaturation of vehicles, average length of ZoI increased by 55, 22 and $21 \%$ at $\mathrm{TI}_{4 \mathrm{w}-\mathrm{nb}}, \mathrm{TI}_{3 \mathrm{w}-\mathrm{nb}}$ and $\mathrm{TI}_{3 \mathrm{w}-\mathrm{wb}}$, respectively, compared with stop- and go-driving conditions. Within average length of ZoI in stop- and godriving conditions, PNCs followed a three degree polynomial form at all TIs. Dimensional analysis suggested that coefficients of polynomial equations at both fourway and three-way TIs were mainly influenced by delay time, wind speed and particle number flux. The PMF analysis suggested that deceleration contributed the most to total

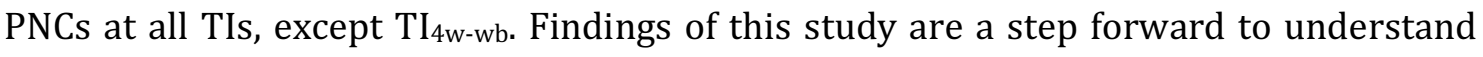
the contribution of different driving conditions towards the total PNCs and their exposure at the TIs.

Keywords: Particle number concentration; Number size distribution; Traffic intersections; Zone of influence; PNC profile; Driving condition; Positive matrix factorisation

\section{Introduction}

Signalised traffic intersections (TIs) are considered as a hotspot of particle number concentration (PNCs). Over 99\% of PNCs are represented by particles below $300 \mathrm{~nm}$ in diameter (Kumar et al., 2011; Kumar et al., 2010). Short-term exposure (i.e. exposure to peak PNCs averaged over short durations such as $1 \mathrm{~s}$ averaged PNC) at the TIs contribute disproportionately higher exposure compared with those experienced at the rest of a commuting route with free-flow traffic conditions. For instance, our recent work found that as little as about $2 \%$ of commuting time spent in car at TIs can contribute up to about 25\% of commuting exposure (Goel and Kumar, 2015). Furthermore, epidemiological studies have shown that even a short exposure of healthy people to traffic-emitted nanoparticles can cause reduction in brain plasticity (Bos et Cite this article as: Goel, A., Kumar, P., 2015. Zone of influence for particle number concentrations at signalised traffic intersections, Atmospheric Environment 123, 25-38. Online Link: http://dx.doi.org/10.1016/j.atmosenv.2015.10.054 
al., 2011) and induce changes in biomarkers of pulmonary and systematic inflammation of healthy individuals (Jacobs et al., 2010).

To assess the effect of changes in driving condition of a vehicle due to traffic signal on PNC, a number of studies are carried out at the fixed monitoring sites around the TIs (Holmes et al., 2005; Morawska et al., 2004; Tsang et al., 2008; Wang et al., 2008). For instance, Tsang et al. (2008) assessed the pedestrian exposure to PNCs at a busy TI in Mong Kok, Hong Kong. They observed a sharp increase in PNCs as a result of vehicle acceleration after about $3 \mathrm{~s}$ when the traffic signal colour changed from red to green. Wang et al. (2008) found that average PNCs at a TI during red-light period was nearly 5-times higher compared to those during green-light period. Aforementioned studies were conducted at a distance of 3 to $5 \mathrm{~m}$ away from the intersecting roads and PNCs has been found to decrease exponentially at distances perpendicular to the road (AlDabbous and Kumar, 2014; Fujitani et al., 2012). Therefore, these studies did not capture the actual on-road PNCs. Moreover, most of past studies are carried out at fixed sites at a point near a TI that made it challenging to capture the on-road profile of PNCs on intersecting roads and at longitudinal distances from the centre of TIs. A number of studies have also carried out mobile monitoring of nanoparticles within the vehicles (Goel and Kumar, 2015; Hudda et al., 2011; Joodatnia et al., 2013a, b; Knibbs et al., 2010; Zhu et al., 2007), but studies focusing on the on-road profiles of PNCs around TIs are still scarce and covered as a part of this study.

There is a certain longitudinal distance along the road at both sides from the centre a TI that experiences elevated level of exhaust emissions due to interruptions in traffic flow produced at the traffic signals. We refer this affected longitudinal length of the road as a zone of influence (ZoI) of a TI. The pollutant concentration in this zone can be many times higher as compare to rest of the route. For instance, Kim et al. (2014) observed that ZoI of a four-way TI for oxides of nitrogen (NOx) extends from -200 to $200 \mathrm{~m}$ distance from the centre of a TI in stop- and go-driving conditions. They found about 200 to $1000 \mathrm{ppb}$ of additional NOx was observed within the ZoI compared with the rest of the route length. This is currently unknown whether the similar increase in PNCs can be expected within a ZoI of a TI. Our recent work suggested up to 29-times higher PNCs at TIs than those found on the rest of the route during free-flow traffic conditions (Goel Cite this article as: Goel, A., Kumar, P., 2015. Zone of influence for particle number concentrations at signalised traffic intersections, Atmospheric Environment 123, 25-38. Online Link: http://dx.doi.org/10.1016/j.atmosenv.2015.10.054 
and Kumar, 2015). This indicates much higher PNCs at different types of TIs but what is the ZoI under different driving conditions is yet poorly understood - this is one of the aims of this study.

As highlighted in our recent review (Goel and Kumar, 2014), dispersion modelling of nanoparticles at TIs is challenging due to a complex interplay among emission, dispersion and transformation processes. A number of operational air quality models addressing the dispersion of gaseous pollutants and particulate matter (PM) at TI are currently available, as seen from the review of related modelling studies in Table 1. However, no such dispersion model exists for the PNCs which could be used at the TIs. As a first step in this direction, one of the aims of this study is to derive a set of equations representing PNC profiles within ZoI of four different types of TIs under frequently occurring stop- and go-driving conditions.

The distinctive features that aim to fill the existing research gaps of this work are as follows. Firstly, as opposed to previous studies (Holder et al., 2014; Hudda et al., 2011; Knibbs et al., 2010; Zhu et al., 2007) that have analysed the effect of velocity variations, ventilation settings and traffic conditions on in-cabin PNCs at individual commuting routes, this study has assessed the effect of traffic driving conditions on on-road PNC profiles at urban traffic hotspots (i.e., TIs). Secondly, this is for the first time when the ZoI of four different types of TIs under varying driving conditions (i.e. stop and go, multiple stopping, and free-flow) are defined for the PNCs. Thirdly, a set of equations representing the PNC profiles at different types of TIs are derived. The coefficients of these equations are represented in the form of parameters such as delay time, particle number flux, wind speed and driving speed. These parameters are chosen from the set of parameters on the basis of dimensional analysis. To perform dimensional analysis, a combination having dimensions similar to the dimensions of the coefficient of generalised PNC profiles is formed. Afterwards $R^{2}$ value was estimated between the derived value of coefficient and the values predicted by using the above-noted parameters. If $\mathrm{R}^{2}$ value was less than 0.5 , we discarded the proposed combination and tried other combinations. This process is explained in detail in in Supplementary Information (SI) Section S1. Such equations could be useful for developing dispersion models for nanoparticles at TIs. Fourthly, this is the first time when positive matrix Cite this article as: Goel, A., Kumar, P., 2015. Zone of influence for particle number concentrations at signalised traffic intersections, Atmospheric Environment 123, 25-38. Online Link: http://dx.doi.org/10.1016/j.atmosenv.2015.10.054 
factorisation (PMF) has been applied to quantify the contribution of PNCs towards the total PNCs released during deceleration, acceleration, cruising and creep-idling at different types of TIs, as defined in Table 2. Such a quantification is useful to plan mitigation strategies to limit emissions and exposure to PNCs at pollution hotspots.

The overall aim of this study is to estimate ZoI and derive a set of equations describing PNC profiles within the ZoI at different types of TIs. Receptor modelling tool, PMF, has been applied to estimate the contribution of different driving conditions towards the total PNCs measured at the TIs and the rest of the route.

\section{Methodology}

\subsection{Route characteristics}

Measurements were conducted on a $6 \mathrm{~km}$ long round route, which included 10 signalised TIs in Guildford, UK (Figure 1). The route was chosen with an intention to pass through a maximum number of TIs of different geometries and built-up area around them so that varying impact of PNC dispersion at these TIs can be assessed (Goel and Kumar, 2014, 2015; Patton et al., 2014). The pavement of each of the studied 10 TIs was made of bituminous flexible pavement, which contained 95\% of asphalt and the remaining contents being filler and bituminous binder (Kumar et al., 2013). Since route had various links, the number of lanes on roads varied from two to four in each direction. Some of the roads have traffic only in one direction (e.g. $\mathrm{TI}_{10}$ and $\mathrm{TI}_{8}$; see Table 3) and the width of each lane was $\sim 3.0 \mathrm{~m}$. Based on number of roads intersecting at a TI, these were divided broadly in two major types: (i) four-way and (ii) three-way TI (Table 3). On the studied route, five of these TIs ( $\mathrm{TI}_{1}, \mathrm{TI}_{2}, \mathrm{TI}_{4}, \mathrm{TI}_{6}$ and $\mathrm{TI}_{10}$ ) were fourway and the rest were three-way. Based on built-up area around a TI, these TIs were further divided into: (i) four-way TI with no built-up area ( $\mathrm{TI}_{4 \mathrm{w}-\mathrm{nb}}$ ), (ii) four-way TI with built-up area $\left(\mathrm{TI}_{4 \mathrm{w} \text {-wb }}\right)$, (iii) three-way TI with no built-up area $\left(\mathrm{TI}_{3 \mathrm{w}-\mathrm{nb}}\right)$, and (iv) three-way TI with built-up area $\left(\mathrm{TI}_{3 \mathrm{w} \text {-wb }}\right)$. Here, TIs with built-up area were assumed to be those TIs that were located in a street canyon with continuous rows of buildings on both sides with an aspect ratio of 0.8 to 0.9 (Table 3). TIs with no built-up area were assumed to be those TIs that were surrounded by residential or commercial buildings but these buildings with height from 6 to $12 \mathrm{~m}$ were placed far apart.

Cite this article as: Goel, A., Kumar, P., 2015. Zone of influence for particle number concentrations at signalised traffic intersections, Atmospheric Environment 123, 25-38. Online Link: http://dx.doi.org/10.1016/j.atmosenv.2015.10.054 
Table 1. Review of available dispersion models for gaseous pollutants and particulate matter with diameter below 2.5 and $10 \mu \mathrm{m}$ (i.e., $\mathrm{PM}_{2.5}$ and $\mathrm{PM}_{10}$, respectively) at the TIs.

\begin{tabular}{lll}
\hline Study & $\begin{array}{l}\text { Pollutant } \\
\text { of focus }\end{array}$ & Description \\
\hline Dirks et al. & CO & The study used a semi-empirical box model to predict CO
\end{tabular}
(2003) concentration at a TI in Hamilton, New Zealand. The correlation coefficient, root mean square error, and mean absolute error for the summer season were $0.7,0.21$, and $0.15 \mathrm{mg} \mathrm{m}^{-3}$, respectively, while the equivalent results for the autumn season were $0.7,0.65$, and $0.43 \mathrm{mg} \mathrm{m}^{-3}$, respectively, when using weekend data to predict for weekdays. The fraction of two (FAC2) (the fraction of data for which the ratio of the predicted to observed concentrations fall within 0.5 and 2) are 0.78 and 0.73 for the summer and autumn seasons, respectively.

\begin{tabular}{|c|c|c|}
\hline $\begin{array}{l}\text { Mishra and } \\
\text { Padmanabhamu } \\
\text { try (2003) }\end{array}$ & $\mathrm{Pb}$ & $\begin{array}{l}\text { This study used California Line source model (CALINE3), California } \\
\text { Line Source for Queuing and Hot Spot Calculation (CAL3QHC) and } \\
\text { Highway Vehicle Particulate Emission Modelling Software (PART5) } \\
\text { to predict ground level lead concentration at five TIs in Delhi, India. } \\
\text { They found CAL3QHC was most suitable for predictions at TIs. }\end{array}$ \\
\hline $\begin{array}{l}\text { Gokhale and } \\
\text { Khare (2005) }\end{array}$ & $\mathrm{CO}$ & $\begin{array}{l}\text { This study used the hybrid modelling approach by combining the } \\
\text { General Finite Line Source Model (GFLSM) with log logistics } \\
\text { distribution to predict carbon monoxide concentrations at ITO TI in } \\
\text { Delhi, India. The validation results showed that the model can } \\
\text { predict CO concentrations fairly well }(\mathrm{d}=0.91 \text { ) in } 10-95 \text { percentile } \\
\text { range. }\end{array}$ \\
\hline $\begin{array}{l}\text { Gokhale and } \\
\text { Raokhande } \\
\text { (2008) }\end{array}$ & $\begin{array}{l}\mathrm{PM}_{10} \text { and } \\
\mathrm{PM}_{2.5}\end{array}$ & $\begin{array}{l}\text { This study evaluated the performance of three air quality models } \\
\text { namely modified General Finite Line Source Model (M-GFLSM), } \\
\text { CALINE-3 and CAL3QHC model at busiest TI in the city of Guwahati, } \\
\text { India. They found that CAL3QHC can make better predictions a } \\
\text { compared to other two models for varied meteorology and traffic } \\
\text { conditions. }\end{array}$ \\
\hline $\begin{array}{l}\text { Oladnia et al. } \\
(2008)\end{array}$ & $\mathrm{CO}$ & $\begin{array}{l}\text { This study predicted CO concentration at T-type TI in Tehran, Iran } \\
\text { using Hybrid Roadway Model (HYROAD). Good agreement between } \\
\text { predicted and monitored concentrations with } \mathrm{R}^{2}=0.83 \text { and } 0.69 \text { for } \\
\text { summer and winter season, respectively was found. }\end{array}$ \\
\hline He et al. (2009) & $\mathrm{PM}_{10}$ & $\begin{array}{l}\text { This study used a semi-empirical box model that includes emission } \\
\text { rates dependent on instantaneous velocity and acceleration of } \\
\text { vehicle at a busy TI in Mong Kok, Hong Kong. They found that the } \\
\text { model performance was quite close to measurement data with index } \\
\text { of agreement ranging from } 0.7 \text { to } 0.84\end{array}$ \\
\hline $\begin{array}{l}\text { Batterman et al. } \\
(2010)\end{array}$ & $\begin{array}{l}\mathrm{CO} \text { and } \\
\mathrm{PM}_{2.5}\end{array}$ & $\begin{array}{l}\text { This study predicted } \mathrm{CO} \text { and } \mathrm{PM}_{2.5} \text { concentrations in a dense } \\
\text { receptor grid over a } 1 \mathrm{~km}^{2} \text { area around the TI of two major roads in } \\
\text { Detroit, Michigan using reduced form of MOBILE } 6.2 \text { for emission } \\
\text { estimates and CALINE } 4 \text { for dispersion modelling. For CO, their } \\
\text { model showed reasonable agreement with annual average and 24- } \\
\text { hour measurement. }\end{array}$ \\
\hline $\begin{array}{l}\text { Ritner et al. } \\
(2013)\end{array}$ & $\mathrm{CO}$ & $\begin{array}{l}\text { In CAL3QHC, only two types of links are defined at TIs (i.e. idling and } \\
\text { free- flow). This study proposed a new method of dividing links by } \\
\text { increments of speed change at the start and end point of link to take } \\
\text { in to account the effect of driving changes on pollutant emissions at } \\
\text { TIs. }\end{array}$ \\
\hline
\end{tabular}

Cite this article as: Goel, A., Kumar, P., 2015. Zone of influence for particle number concentrations at signalised traffic intersections, Atmospheric Environment 123, 25-38. Online Link: http://dx.doi.org/10.1016/j.atmosenv.2015.10.054 
Table 2. Description of deceleration, acceleration, cruising and creep-idling conditions.

\begin{tabular}{lll}
\hline Driving conditions & $\begin{array}{l}\text { Driving speed }\left(u ; \mathrm{km} \mathrm{h}^{-}\right. \\
1)\end{array}$ & $\begin{array}{l}\text { Rate of change of driving speed (i.e. } \\
\left.d u / d t ; \mathrm{m} \mathrm{s}^{-2}\right)\end{array}$ \\
\hline Deceleration & $>2$ & $<0$ \\
\hline Acceleration & $>2$ & $>0$ \\
\hline Cruising & $>2$ & 0 \\
\hline Creep-idling & $<2$ & $>-2$ and $<2$ \\
\hline
\end{tabular}

Average daily traffic flow on different roads intersecting these TIs was obtained from the DoT (2013). Total traffic volume at a TI was estimated by summing the traffic flow on each of the roads intersecting at a $\mathrm{TI} \mathrm{TI}_{8}$ and $\mathrm{TI}_{7}$ caters to highest traffic flow of 160824 veh day ${ }^{-1}$ while it was lowest (9846 veh day-1) at the $\mathrm{TI}_{3}$. Further details of traffic volume and the TIs are presented in Table 3.
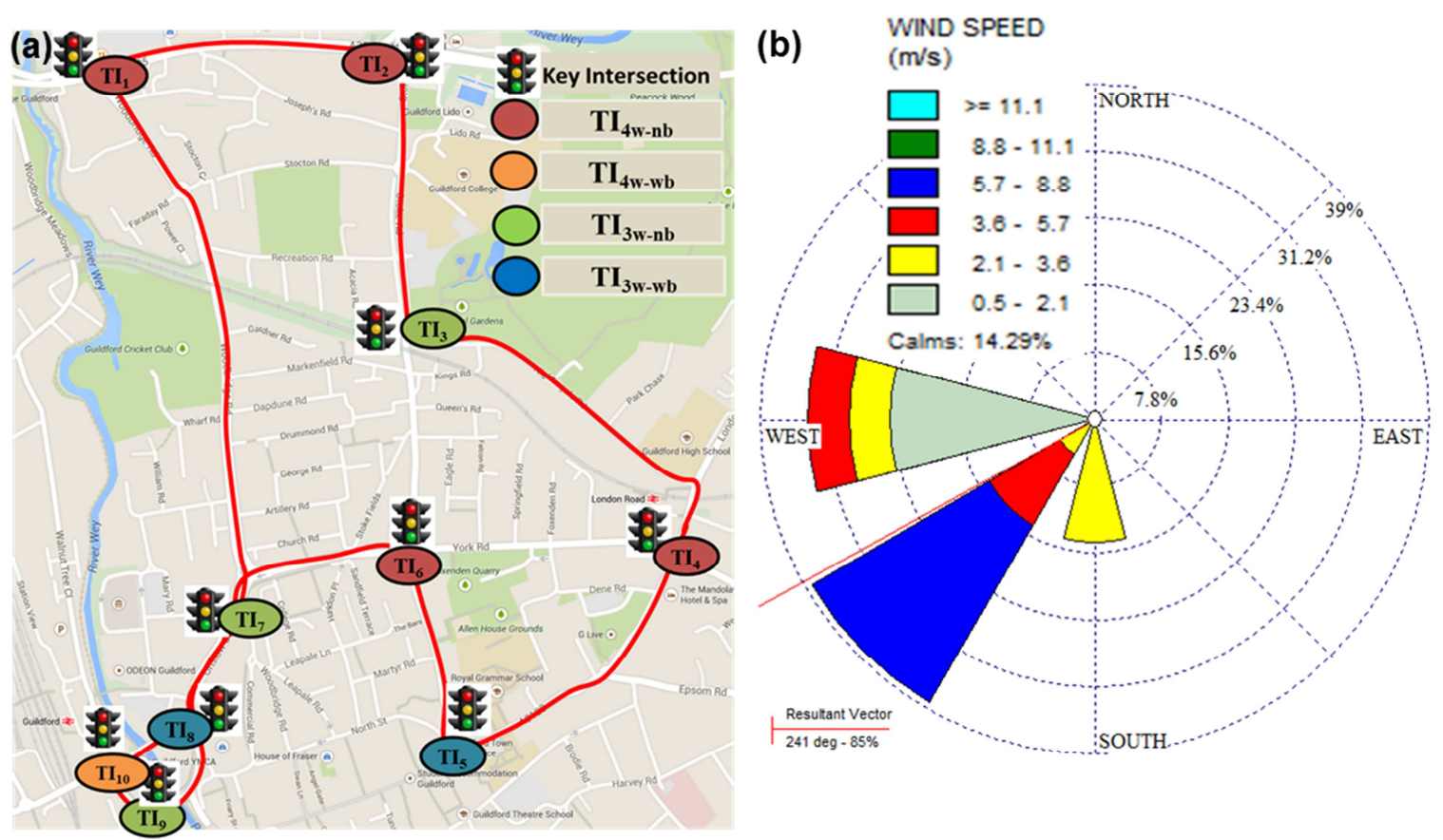

Figure. 1 (a) Map of the study route showing the major TIs, and (b) wind rose diagram for the monitoring period.

Cite this article as: Goel, A., Kumar, P., 2015. Zone of influence for particle number concentrations at signalised traffic intersections, Atmospheric Environment 123, 25-38. Online Link: http://dx.doi.org/10.1016/j.atmosenv.2015.10.054 


\subsection{Study design}

The measurements were conducted inside the car cabin with windows fully open during morning and evening rush hours between 4 and 7 April 2014. Surface area of each of the four windows was $0.275 \mathrm{~m}^{2}$, giving total area of all windows as $1.10 \mathrm{~m}^{2}$. The tyres of the experimental car were made of carbon based materials ( 74\%), metal (16.5\%), textiles (5.5\%) and additives (4\%). Experimental car was equipped with front disc brake and rear drum braking system. The route was driven from North to South (NS) and South to North (S-N) directions during morning and evening rush hours, respectively (Figure 1). A total of 22 runs were made; 12 of them were made during morning hours and the rest during the evening hours. Since the measurements were made close to the window that were fully open, these measurements were assumed equivalent to on-road measurements. This assumption is justified based on the fact that only about $2 \%$ differences were noted between the average PNCs inside and outside the car when all windows were fully open and measurements were made on backseat of the car near the window (Goel and Kumar, 2015). The meteorological data during the measurements was collected from weather station located at the Heathrow airport (Metoffice, 2014). During the measurement period, average wind speed, temperature and relative humidity was found to be $3 \pm 3 \mathrm{~m} \mathrm{~s}^{-1}, 9 \pm 3{ }^{\circ} \mathrm{C}$ and $73 \pm 3 \%$, respectively.

\subsection{Instrumentation}

A diesel-fuelled car (Ford Fiesta; 2002 registration; $1400 \mathrm{cc}$ ) was used for the study. During experiments, there were three non-smoking occupants in the car including the driver. The experimental car was equipped with the filter-fitted ventilation and heating systems. A Cambustion DMS50 was deployed to measure particle number distribution (PNDs) in the 5-560 $\mathrm{nm}$ size range at a sampling rate of 10 Hz. Details of the working principle, detection efficiencies and noise levels can be seen elsewhere(Al-Dabbous and Kumar, 2014; Carpentieri and Kumar, 2011; Goel and Kumar, 2015). Position and speed of vehicle was continuously recorded on a second basis (i.e. $1 \mathrm{~Hz}$ ) using a Global Positioning System (GPS; Garmin Oregon 350). Panasonic HC-V500 camera was positioned on the dashboard of the car to take traffic videos at the time of measurements. Timestamps of all the instruments were matched in the beginning of the experiment.

Cite this article as: Goel, A., Kumar, P., 2015. Zone of influence for particle number concentrations at signalised traffic intersections, Atmospheric Environment 123, 25-38. Online Link: http://dx.doi.org/10.1016/j.atmosenv.2015.10.054 


\subsection{Data acquisition}

A total of 2,88,000 data points of size-resolved PNDs during $8 \mathrm{~h}$ of measurements at a sampling frequency of $10 \mathrm{~Hz}$ were collected over the studied runs. These data were then analysed by Microsoft excel with the use of DMS50 data processing tools. GPS data was converted from .gpx to excel file with the help of mapsource software. Centre of each of the 10 TIs was identified by plotting the route on Google earth. For each of the 22 runs, driving speed profile of an experimental car was plotted at all the 10 TIs to identify the driving conditions during the individual runs. Based on the driving condition of experimental vehicle at each of the TI, all the runs were further categorised in three categories: (i) stop and go, (ii) multiple stopping, and (iii) free-flow driving conditions (Table 4).

Baseline PNCs for each run were derived by taking a 30 s rolling average of the lowest $5^{\text {th }}$ percentile value of the 1 s concentration time series (Hudda et al., 2014). This methodology was adopted to smooth the data and to exclude the impact of micro-scale. To avoid potential sampling of self-exhaust from the experimental vehicle, we censored about $5 \%$ of the total data for situations when wind was approaching from the rear of the experimental vehicle and driving speed was less than $2 \mathrm{~km} \mathrm{~h}^{-1}$.

\subsection{Estimation of ZoI}

In order to estimate the ZoI in longitudinal direction of the road, PNC data for $\pm 200 \mathrm{~m}$ distance from the centre of each of the TI was extracted. This preliminary distance was chosen with an assumption that the effects of traffic lights on emissions dies back to normal during free-flow traffic conditions by the end of $200 \mathrm{~m}$ in each direction. This assumption is based on our field observations, where we have observed an average driving speed less than $20 \mathrm{~km} \mathrm{~h}^{-1}$ within $\pm 200 \mathrm{~m}$ and greater than $25 \mathrm{~km} \mathrm{~h}^{-1}$ beyond $200 \mathrm{~m}$. To estimate the ZoI, driving speed and PNC profiles for all the 22 runs at each of the ten TIs were plotted with respect to changing distance from the centre of a $\mathrm{TI}$, resulting in a total of 220 profiles.

We assumed that the ZoI is a length of a road section between points of intersections around a TI where driving speed and PNC profiles intersect each other, as seen in Figure 3. ZoI represents drop in driving speed with a corresponding rise in PNC (Figure 3).

Cite this article as: Goel, A., Kumar, P., 2015. Zone of influence for particle number concentrations at signalised traffic intersections, Atmospheric Environment 123, 25-38. Online Link: http://dx.doi.org/10.1016/j.atmosenv.2015.10.054 
First and second points of the juncture in the driving direction of experimental car are defined as $X_{1}$ and $X_{2}$, respectively (Figure 3). The values of $X_{1}$ and $X_{2}$ were tabulated in the Supplementary Information (SI) Tables S1 and S2 for each of the 10 TIs during different driving conditions and driving directions shown in Figure 2.

Table 3. Description of TIs on the study route.

\begin{tabular}{|c|c|c|c|c|c|}
\hline $\begin{array}{l}\text { TI } \\
\text { number }\end{array}$ & $\begin{array}{l}\text { Number of roads } \\
\text { intersecting at a } \\
\text { TI }\end{array}$ & $\begin{array}{l}\text { Built-up area around a } \\
\text { TI }\end{array}$ & $\begin{array}{l}\text { Average daily } \\
\text { traffic (veh } \\
\text { day }^{-1} \text { ) }\end{array}$ & $\begin{array}{l}\text { Main road } \\
\text { characteristic }\end{array}$ & $\begin{array}{l}\text { Pavement } \\
\text { characteristics }\end{array}$ \\
\hline $\mathrm{TI}_{1}$ & 4 & $\begin{array}{l}2-3 \text { building with } \\
\text { average building } \\
\text { height of } \sim 6 \mathrm{~m}\end{array}$ & 81066 & $\begin{array}{l}\text { All } 4 \text { roads with } \\
\text { 2-way traffic }\end{array}$ & \multirow{10}{*}{$\begin{array}{c}\text { All ten TIs } \\
\text { were having } \\
\text { bituminous } \\
\text { flexible } \\
\text { pavement } \\
\text { comprised of } \\
\text { 95\% asphalt } \\
\text { and } 5 \% \text { filler } \\
\text { and } \\
\text { bituminous } \\
\text { binder }\end{array}$} \\
\hline $\mathrm{TI}_{2}$ & 4 & No buildings & 45104 & $\begin{array}{l}\text { All } 4 \text { roads with } \\
\text { 2-way traffic }\end{array}$ & \\
\hline $\mathrm{TI}_{3}$ & 3 & $\begin{array}{l}\text { Residential area with } \\
\text { average height of } \\
\text { buildings } \sim 6 \mathrm{~m}\end{array}$ & 9846 & $\begin{array}{l}\text { All } 3 \text { roads with } \\
\text { 2-way traffic }\end{array}$ & \\
\hline $\mathrm{TI}_{4}$ & 4 & $\begin{array}{l}\text { Residential area with } \\
\text { average height of } \\
\text { buildings } \sim 6 \mathrm{~m}\end{array}$ & 143508 & $\begin{array}{l}\text { All } 4 \text { roads with } \\
2 \text {-way traffic }\end{array}$ & \\
\hline $\mathrm{TI}_{5}$ & 3 & $\begin{array}{l}\text { Surrounded by a street } \\
\text { canyon with an aspect } \\
\text { ratio } \sim 0.8 \text { and average } \\
\text { height of buildings } \\
\text { around a TI was } \sim 9 \mathrm{~m}\end{array}$ & 56284 & $\begin{array}{l}\text { All } 3 \text { roads with } \\
2 \text {-way traffic }\end{array}$ & \\
\hline $\mathrm{TI}_{6}$ & 4 & $\begin{array}{l}\text { Residential area with } \\
\text { average height of } \\
\text { buildings } \sim 6 \mathrm{~m}\end{array}$ & 103680 & $\begin{array}{l}\text { All } 4 \text { roads with } \\
2 \text {-way traffic }\end{array}$ & \\
\hline $\mathrm{TI}_{7}$ & 3 & $\begin{array}{l}\text { Commercial area with } \\
\text { average height of } \\
\text { buildings } \sim 8 \mathrm{~m}\end{array}$ & 160824 & $\begin{array}{l}\text { All } 3 \text { roads with } \\
\text { 2-way traffic }\end{array}$ & \\
\hline $\mathrm{TI}_{8}$ & 3 & $\begin{array}{l}\text { Surrounded by a street } \\
\text { canyon with an aspect } \\
\text { ratio of } \sim 0.8 \text { and } \\
\text { average height of } \\
\text { buildings around a TI } \\
\text { was } \sim 12 \mathrm{~m}\end{array}$ & 160824 & $\begin{array}{l}2 \text { roads with } 2 \text { - } \\
\text { way and } 1 \text { with } \\
1 \text {-way traffic }\end{array}$ & \\
\hline $\mathrm{TI}_{9}$ & 3 & $\begin{array}{l}2-3 \text { building with } \\
\text { average building } \\
\text { height of } \sim 6 \mathrm{~m}\end{array}$ & - & $\begin{array}{l}2 \text { roads with } 1 \text { - } \\
\text { way and } 1 \text { with } \\
2 \text {-way traffic }\end{array}$ & \\
\hline $\mathrm{TI}_{10}$ & 4 & $\begin{array}{l}\text { Surrounded by a street } \\
\text { canyon with an aspect } \\
\text { ratio } \sim 0.9 \text { and average } \\
\text { height of buildings } \\
\text { around a TI was } \sim 12 \mathrm{~m}\end{array}$ & 107784 & $\begin{array}{l}2 \text { roads with } 2 \text { - } \\
\text { way and } 2 \\
\text { roads with } 1 \text { - } \\
\text { way traffic }\end{array}$ & \\
\hline
\end{tabular}

Cite this article as: Goel, A., Kumar, P., 2015. Zone of influence for particle number concentrations at signalised traffic intersections, Atmospheric Environment 123, 25-38. Online Link: http://dx.doi.org/10.1016/j.atmosenv.2015.10.054 


\section{$2.6 \quad$ PMF modelling}

PMF is a receptor model that has been used in numerous air pollution studies to identify the sources and apportioning their contribution (Al-Dabbous and Kumar., 2015; Kumar et al., 2013; Harrison et al., 2011). Unlike other receptor models such as principal component analysis (PCA), it takes into account the standard deviations of observed data values and put the constraint of non-negativity of all the factor matrices and elements in order to have physically meaningful solutions. It is thus a weighted least square problem in which a certain number of factors have to be determined in order to minimize an 'object function'. Thus, the PMF model can provide inherently better understanding of the data than eigenvector-based methods (i.e. PCA). Moreover, it allows weighing of individual data points. Due to individual weighing of data ponts, problematic data such as outliers or below-detection limit can be entered into the model with appropriated weight, avoiding rejection of such data (JRC, 2009).

PMF analysis is carried out using the US EPA's PMF model (version 5.0) for quantifying the contribution of traffic-produced PNCs during deceleration, creep-idling, acceleration and cruising towards the total PNCs measured at the TIs and the rest of the study route. The input dataset composed 32 variables. These variables included PNDs in 32 size classes covering a size range of 5-560 $\mathrm{nm}$. The uncertainty data file supplied by the instrument manufacturer (Cambustion Ltd., Cambridge), consisting of size-specific minimum detection limits and error fractions, was also included in the PMF (AlDabbous and Kumar, 2015). Unlike Domínguez-Sáez et al. (2012) who used median PNDs curve on sub-sections of their study route to prepare input data for PMF, we used the run-wise data related to the study route. We performed a sample run of PMF by arranging input data using the following different approaches. Firstly, we averaged time series PND data of all runs since the total duration of each run was nearly similar

(960 \pm 240 s). Secondly, we run PMF model separately for 22 individual runs and then taking up average of resulted source profiles. Lastly, we stacked up run-wise PND data on top of each other. All the three approaches resulted in similar source profiles and therefore we selected the third approach to arrange input data for PMF since this approach enabled us to compare the time series of normalised contribution of each of the factor with variations in driving speed. This comparison played an important role in assigning different factors to different driving conditions.

Cite this article as: Goel, A., Kumar, P., 2015. Zone of influence for particle number concentrations at signalised traffic intersections, Atmospheric Environment 123, 25-38. Online Link: http://dx.doi.org/10.1016/j.atmosenv.2015.10.054 

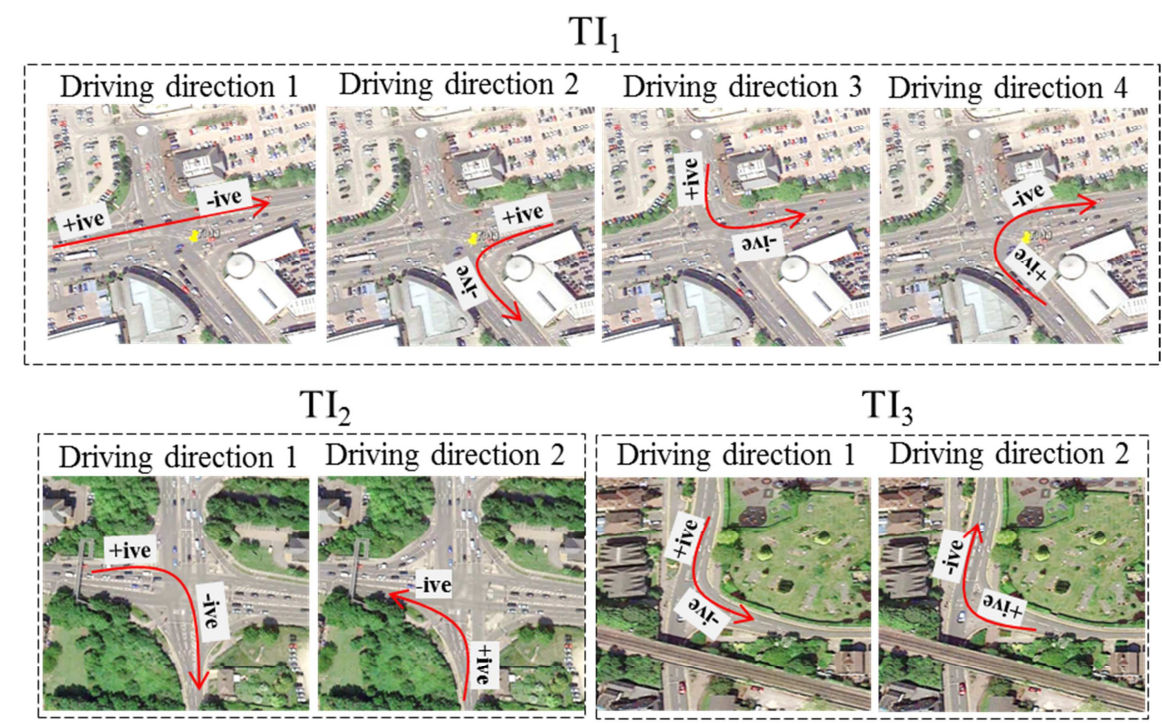

$\mathrm{TI}_{4}$

$\mathrm{TI}_{3}$
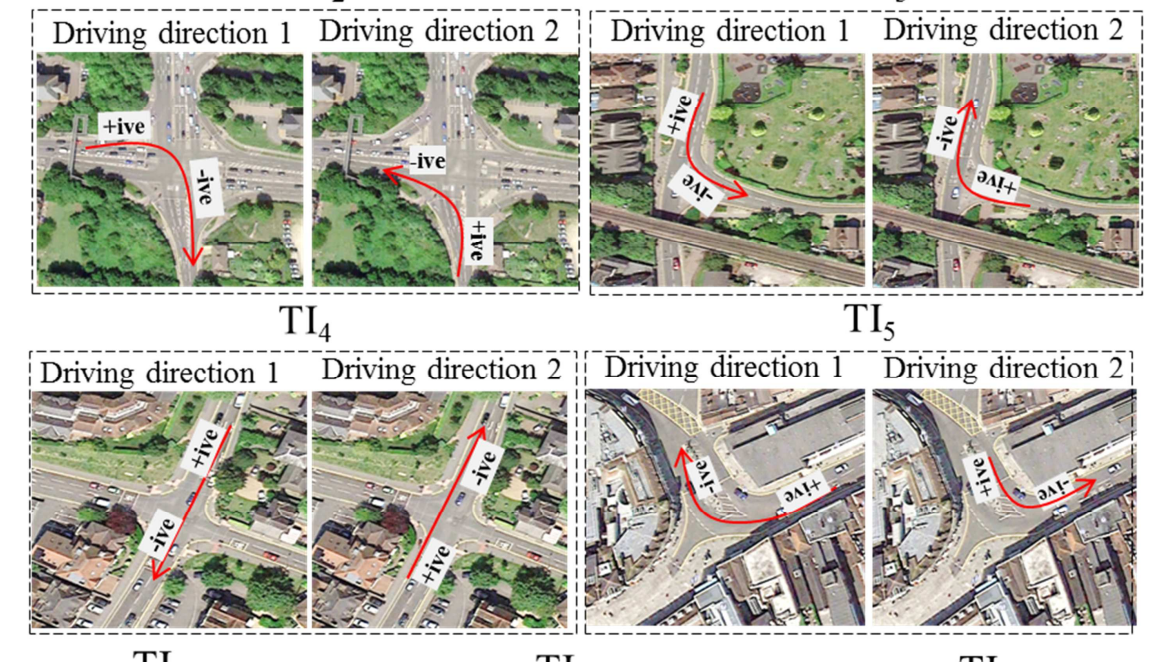

$\mathrm{TI}_{5}$

$\mathrm{TI}_{6}$

$\mathrm{TI}_{7}$

$\mathrm{TI}_{8}$

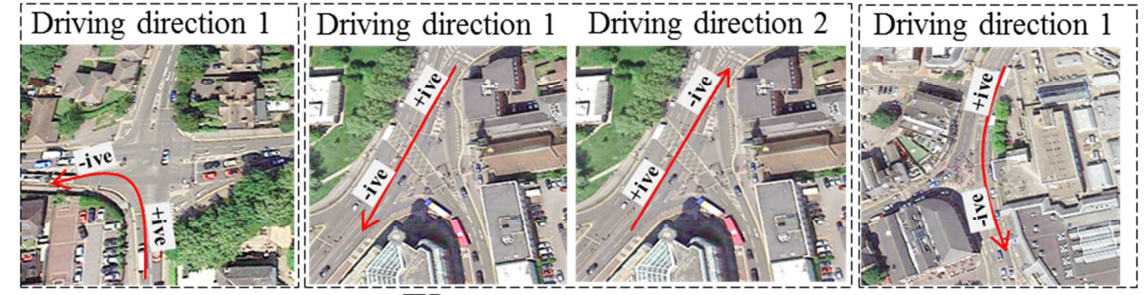

$\mathrm{TI}_{9}$

$\mathrm{TI}_{10}$

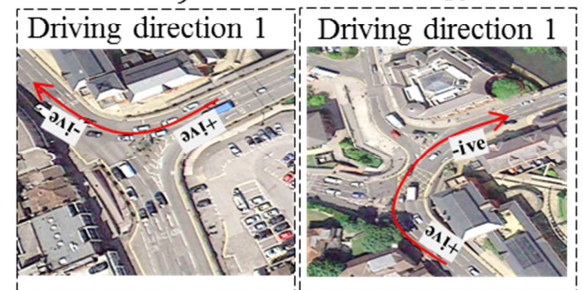

Figure. 2. Google image of driving directions covered by an experimental vehicle at each of the 10 TIs. Arrows represent driving directions of our experimental vehicle; tail and head of the arrow represents driving direction from '+' and '-' at each TI.

Cite this article as: Goel, A., Kumar, P., 2015. Zone of influence for particle number concentrations at signalised traffic intersections, Atmospheric Environment 123, 25-38. Online Link: http://dx.doi.org/10.1016/j.atmosenv.2015.10.054 


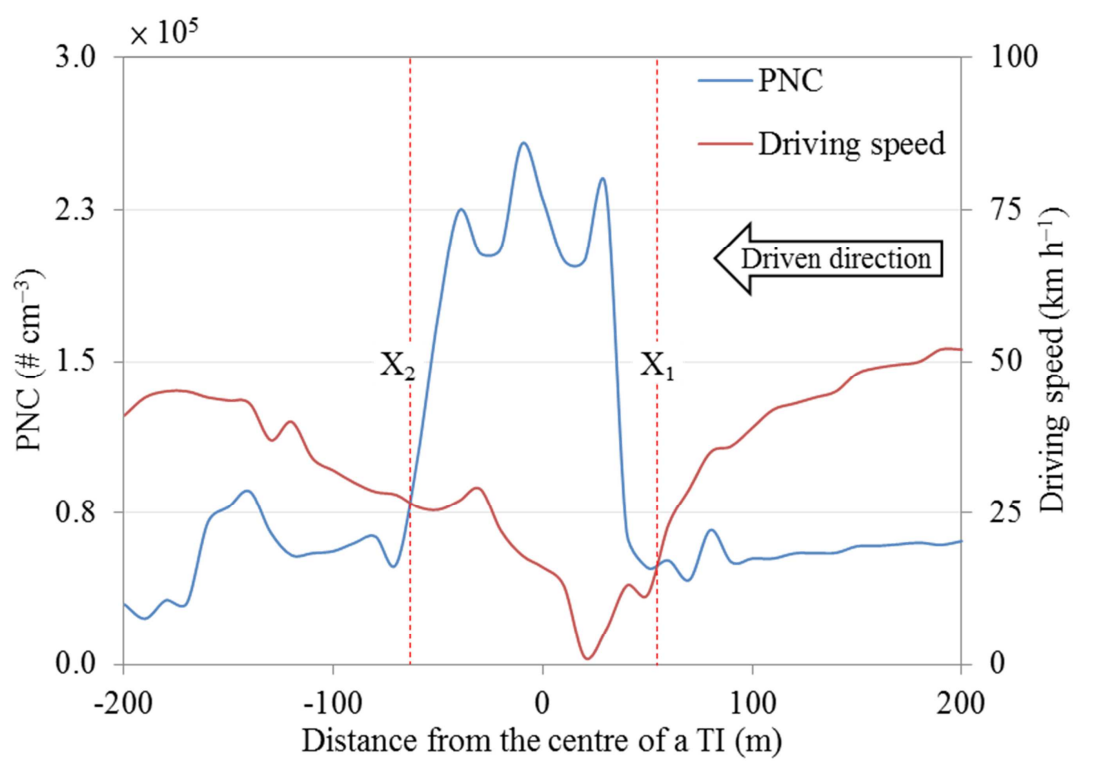

Figure. 3. An example showing the ZoI during run 11 at $\mathrm{TI}_{4}$, where on-road PNCs and driving speed profiles intersect each other at points where PNCs are increasing and driving speed is decreasing. The distance between the intersecting points $X_{1}$ and $X_{2}$ from the centre of a TI is taken as a measure of ZoI. Arrow represents the driving direction of our experimental vehicle.

\section{Results and discussions}

Firstly we quantified the longitudinal distance of ZoI for PNCs at four different types of TIs (i.e. $\mathrm{TI}_{4 \mathrm{w}-\mathrm{nb}}, \mathrm{TI}_{4 \mathrm{w}-\mathrm{wb}}, \mathrm{TI}_{3 \mathrm{w}-\mathrm{nb}}$ and $\mathrm{TI}_{3 \mathrm{w}-\mathrm{wb}}$ ) under three unique driving conditions. These included stop and go, multiple stopping, and free-flow driving conditions (Section 3.1 to 3.3). Thereafter, PNC profiles within the ZoI of different types of TIs are interpreted based on dimensional analysis (Section 3.4). PMF analysis is then carried out to quantify the contribution of PNCs released by a vehicle during deceleration, creep-idling, acceleration and cruising to total PNCs at the selected TI types and the rest of the route (Section 3.5).

Cite this article as: Goel, A., Kumar, P., 2015. Zone of influence for particle number concentrations at signalised traffic intersections, Atmospheric Environment 123, 25-38. Online Link: http://dx.doi.org/10.1016/j.atmosenv.2015.10.054 


\subsection{Common features of ZoI between stop and go and multiple stopping driving conditions}

Longitudinal distance representing ZoI at the TIs is estimated using the approach explained in Section 2.5.1. ZoI at each of the 10 TIs, separately for each run, were derived for stop and go and multiple stopping driving conditions (SI Table S1 and S2). These runs were then divided in four different TI categories $\left(\mathrm{TI}_{4 \mathrm{w}-\mathrm{nb}}, \mathrm{TI}_{4 \mathrm{w}-\mathrm{wb}}, \mathrm{TI}_{3 \mathrm{w}-\mathrm{nb}}\right.$ and

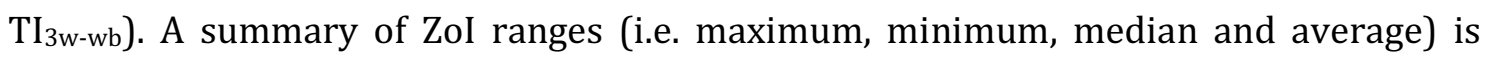
presented in Tables 5 and 6. ZoI represents a length of a road around a TI that is affected by higher particle number emissions as compared to rest of route where freeflow driving conditions persist. Maximum, median and average length of ZoI reported in Table 5 and 6 can be used to serve different purposes depending on individual's choice. For example, maximum length of ZoI at a TI can be considered for dispersion modelling purposes. As it results in conservative estimation of PNCs, and cover all possible receptor locations around a TI that are affected by higher particle number emissions. Maximum length of ZoI was found to be up to 3.6-times the median length, and the median length were within $\pm 5 \%$ of average length of ZoI (Tables 7 and 8). Given that the maximum and median values are most relevant for both the driving conditions, these are chosen for the subsequent discussion throughout the article.

In both the driving conditions, maximum length of $\mathrm{ZoI}$ is highest at $\mathrm{TI}_{4 \mathrm{w}-\mathrm{nb}}$, followed by $\mathrm{TI}_{3 \mathrm{w} \text {-wb, }} \mathrm{TI}_{3 \mathrm{w}-\mathrm{nb}}$ and $\mathrm{TI}_{4 \mathrm{w} \text {-wb }}$ (Tables 7 and 8$)$. A good linear correlation $\left(R^{2}=0.70\right.$ in stop and go and $R^{2}=0.66$ in multiple stopping) is observed between maximum length of ZoI and idling time at a TI. Higher idling found to lead to a longer queue length at the TIs, resulting in longer ZoI.

Unlike the maximum length, median length of ZoI was highest at $\mathrm{TI}_{3 \mathrm{w} \text {-wb }}$, followed by $\mathrm{TI}_{4 \mathrm{w}-\mathrm{nb}}, \mathrm{TI}_{3 \mathrm{w}-\mathrm{nb}}$ and $\mathrm{TI}_{4 \mathrm{w}-\mathrm{wb}}$ (Tables 7 and 8) in both driving conditions. A reasonable good linear correlation $\left(R^{2}=0.55\right)$ was found between median length of ZoI and the average acceleration (Table 7) for stop and go driving condition. However, no such correlation was found for multiple stopping driving condition.

Cite this article as: Goel, A., Kumar, P., 2015. Zone of influence for particle number concentrations at signalised traffic intersections, Atmospheric Environment 123, 25-38. Online Link: http://dx.doi.org/10.1016/j.atmosenv.2015.10.054 
Table 4. Run-wise description of different driving conditions at four different types of TIs. Baseline PNC were estimated based on the approach presented in Section 2.4 .

\begin{tabular}{|c|c|c|c|c|c|c|c|c|c|c|c|c|c|}
\hline \multirow{3}{*}{ Run } & \multicolumn{10}{|c|}{ Driving condition } & \multirow{3}{*}{$\begin{array}{c}\text { Baseline PNC } \\
\left(\# \mathrm{~cm}^{-3}\right) \times \\
10^{4}\end{array}$} & \multirow{3}{*}{$\begin{array}{l}\text { Wind } \\
\text { speed } \\
\left(\mathrm{m} \mathrm{s}^{-}\right. \\
1)\end{array}$} & \multirow{3}{*}{$\begin{array}{c}\text { Wind } \\
\text { direction } \\
\left(^{\circ}\right)\end{array}$} \\
\hline & \multicolumn{4}{|c|}{$\mathrm{TI}_{4 \mathrm{w}-\mathrm{nb}}$} & \multirow{2}{*}{$\begin{array}{c}\mathrm{TI}_{4 \mathrm{w}-\mathrm{wb}} \\
\mathrm{TI}_{10}\end{array}$} & \multicolumn{3}{|c|}{$\mathrm{TI}_{3 \mathrm{w}-\mathrm{nb}}$} & \multicolumn{2}{|c|}{$\mathrm{TI}_{3 \mathrm{w} \text {-wb }}$} & & & \\
\hline & $\mathrm{TI}_{1}$ & $\mathrm{TI}_{2}$ & $\mathrm{TI}_{4}$ & $\mathrm{TI}_{6}$ & & $\mathrm{TI}_{3}$ & $\mathrm{TI}_{7}$ & TI9 & $\mathrm{TI}_{5}$ & $\mathrm{TI} 8$ & & & \\
\hline R1 & Stop-go ${ }^{1,1}$ & Stop-go 2,1 & $\begin{array}{l}\text { Stop- } \\
\text { go }^{4,1}\end{array}$ & $\begin{array}{l}\text { Free- } \\
\text { flow }^{6,1}\end{array}$ & Stop-go $\mathrm{g}^{10,1}$ & $\begin{array}{l}\text { Free- } \\
\text { flow }{ }^{3,1}\end{array}$ & Stop-go ${ }^{7,1}$ & $\begin{array}{c}\text { Multiple } \\
\text { stopping, }\end{array}$ & $\begin{array}{l}\text { Free- } \\
\text { flow }^{5,1}\end{array}$ & Free-flow 8,1 & 5.01 & 0.3 & 270 \\
\hline $\mathrm{R} 2$ & Stop-go $0^{1,2}$ & Free-flow ${ }^{2,1}$ & $\begin{array}{l}\text { Stop- } \\
\text { go }^{4,1}\end{array}$ & $\begin{array}{l}\text { Stop- } \\
\text { go }^{6,1}\end{array}$ & Stop-go $0^{10,1}$ & $\begin{array}{l}\text { Free- } \\
\text { flow }^{3,1}\end{array}$ & $\begin{array}{c}\text { Multiple } \\
\text { stopping }\end{array}$ & $\begin{array}{c}\text { Multiple } \\
\text { stopping }\end{array}$ & $\begin{array}{l}\text { Free- } \\
\text { flow }^{5,1}\end{array}$ & Stop-go ${ }^{8,1}$ & 6.93 & 1.1 & 270 \\
\hline R3 & Stop-go ${ }^{1,2}$ & Free-flow ${ }^{2,2}$ & $\begin{array}{l}\text { Free- } \\
\text { flow }^{4,2}\end{array}$ & - & Free-flow ${ }^{10,1}$ & $\begin{array}{l}\text { Stop- } \\
\text { go }^{3,2}\end{array}$ & Stop-go ${ }^{7,1}$ & Free-flow 9,1 & Stop-go 5,2 & Stop-go ${ }^{8,1}$ & 3.38 & 1.1 & 270 \\
\hline R4 & $\begin{array}{c}\text { Free-flow } \\
1,2\end{array}$ & Stop-go 2,2 & $\begin{array}{c}\text { Stop-go } \\
4,2\end{array}$ & - & Free-flow ${ }^{10,1}$ & $\begin{array}{c}\text { Stop- } \\
\text { go }^{3,2}\end{array}$ & $\begin{array}{c}\text { Multiple } \\
\text { stopping7,1 }\end{array}$ & Stop-go 9,1 & Stop-go 5,2 & Stop-go ${ }^{8,1}$ & 4.01 & 1.1 & 270 \\
\hline R5 & $\begin{array}{c}\text { Free-flow } \\
1,2\end{array}$ & Stop-go ${ }^{2,2}$ & $\begin{array}{c}\text { Stop-go } \\
4,2\end{array}$ & - & Stop-go $0^{10,1}$ & $\begin{array}{l}\text { Stop- } \\
\text { go }^{3,2}\end{array}$ & $\begin{array}{c}\text { Multiple } \\
\text { stopping }\end{array}$ & $\begin{array}{c}\text { Multiple } \\
\text { stopping }\end{array}$ & Stop-go 5,2 & Stop-go $0^{8,1}$ & 4.00 & 1.1 & 270 \\
\hline R6 & $\begin{array}{c}\text { Free-flow } \\
1,2\end{array}$ & $\begin{array}{c}\text { Multiple } \\
\text { stopping } 2,2\end{array}$ & $\begin{array}{l}\text { Multiple } \\
\text { stopping }{ }^{4} \\
, 2\end{array}$ & - & Stop-go ${ }^{10,1}$ & $\begin{array}{l}\text { Stop- } \\
\text { go }^{3,2}\end{array}$ & Free-flow & Stop-go 9,1 & $\begin{array}{l}\text { Free- } \\
\text { flow }^{5,2}\end{array}$ & Stop-go ${ }^{8,1}$ & 0.89 & 7.2 & 225 \\
\hline R7 & Stop-go ${ }^{1,2}$ & $\begin{array}{c}\text { Multiple } \\
\text { stopping2,2 }\end{array}$ & $\begin{array}{l}\text { Multiple } \\
\text { stopping4 } \\
, 2\end{array}$ & - & Free-flow ${ }^{10,1}$ & $\begin{array}{l}\text { Stop- } \\
\text { go }^{3,2}\end{array}$ & $\begin{array}{c}\text { Multiple } \\
\text { stopping }\end{array}$ & Stop-go 9,1 & $\begin{array}{l}\text { Free- } \\
\text { flow }^{5,2}\end{array}$ & Free-flow 8,1 & 1.24 & 7.5 & 225 \\
\hline R8 & Stop-go ${ }^{1,2}$ & $\begin{array}{c}\text { Multiple } \\
\text { stopping } 2,2\end{array}$ & $\begin{array}{c}\text { Stop- } \\
\text { go }^{4,2}\end{array}$ & - & Free-flow ${ }^{10,1}$ & $\begin{array}{l}\text { Free- } \\
\text { flow }^{3,2}\end{array}$ & Stop-go ${ }^{7,1}$ & Free-flow 9,1 & $\begin{array}{l}\text { Free- } \\
\text { flow }^{5,2}\end{array}$ & Stop-go ${ }^{8,1}$ & 1.68 & 6.7 & 225 \\
\hline R9 & $\begin{array}{c}\text { Free-flow } \\
1,2\end{array}$ & $\begin{array}{c}\text { Multiple } \\
\text { stopping } 2,2\end{array}$ & $\begin{array}{l}\text { Stop- } \\
\text { go }^{4,2}\end{array}$ & - & Stop-go $0^{10,1}$ & $\begin{array}{l}\text { Free- } \\
\text { flow }^{3,2}\end{array}$ & Stop-go ${ }^{7,1}$ & Stop-go 9,1 & $\begin{array}{l}\text { Free- } \\
\text { flow } 5,2^{5}\end{array}$ & Free-flow ${ }^{8,1}$ & 1.27 & 6.1 & 225 \\
\hline R10 & $\begin{array}{c}\text { Free-flow } \\
1,2\end{array}$ & Stop-go 2,2 & $\begin{array}{l}\text { Free- } \\
\text { flow }^{4,2}\end{array}$ & - & Stop-go ${ }^{10,1}$ & $\begin{array}{l}\text { Free- } \\
\text { flow }^{3,2}\end{array}$ & Free-flow 7,1 & Free-flow 9,1 & $\begin{array}{c}\text { Multiple } \\
\text { stoppings,2 }\end{array}$ & Free-flow ${ }^{8,1}$ & 1.75 & 6.1 & 225 \\
\hline R11 & Stop-go $0^{1,2}$ & Stop-go ${ }^{2,2}$ & $\begin{array}{l}\text { Stop- } \\
\text { go }^{4,2}\end{array}$ & - & Free-flow ${ }^{10,1}$ & $\begin{array}{l}\text { Free- } \\
\text { flow }^{3,2}\end{array}$ & $\begin{array}{c}\text { Multiple } \\
\text { stopping }\end{array}$ & Stop-go 9,1 & $\begin{array}{l}\text { Free- } \\
\text { flow }^{5,2}\end{array}$ & Free-flow 8,1 & 0.71 & 5.6 & 225 \\
\hline
\end{tabular}

Cite this article as: Goel, A., Kumar, P., 2015. Zone of influence for particle number concentrations at signalised traffic intersections, Atmospheric Environment 123, 2538. Online Link: http://dx.doi.org/10.1016/j.atmosenv.2015.10.054 


\begin{tabular}{|c|c|c|c|c|c|c|c|c|c|c|c|c|c|}
\hline \multirow{3}{*}{ Run } & \multicolumn{10}{|c|}{ Driving condition } & \multirow{3}{*}{$\begin{array}{c}\text { Baseline PNC } \\
\left(\# \mathrm{~cm}^{-3}\right) \times \\
10^{4}\end{array}$} & \multirow{3}{*}{$\begin{array}{l}\text { Wind } \\
\text { speed } \\
\left(\mathrm{m} \mathrm{s}^{-}\right. \\
1)\end{array}$} & \multirow{3}{*}{$\begin{array}{c}\text { Wind } \\
\text { direction } \\
\left(^{\circ}\right)\end{array}$} \\
\hline & \multicolumn{4}{|c|}{$\mathrm{TI}_{4 \mathrm{w}-\mathrm{nb}}$} & \multirow{2}{*}{$\frac{\mathrm{TI}_{4 \mathrm{w}-\mathrm{wb}}}{\mathrm{TI}_{10}}$} & \multicolumn{3}{|c|}{$\mathrm{TI}_{3 \mathrm{w}-\mathrm{nb}}$} & \multicolumn{2}{|c|}{$\mathrm{TI}_{3 \mathrm{w}-\mathrm{wb}}$} & & & \\
\hline & $\mathrm{TI}_{1}$ & $\mathrm{TI}_{2}$ & $\mathrm{TI}_{4}$ & $\mathrm{TI}_{6}$ & & $\mathrm{TI}_{3}$ & $\mathrm{TI}_{7}$ & $\mathrm{TI}_{9}$ & $\mathrm{TI}_{5}$ & $\mathrm{TI}_{8}$ & & & \\
\hline R12 & Stop-go ${ }^{1,3}$ & Stop-go ${ }^{2,1}$ & $\begin{array}{l}\text { Stop- } \\
\text { go }^{4,1}\end{array}$ & $\begin{array}{l}\text { Free- } \\
\text { flow }^{6,1}\end{array}$ & Stop-go ${ }^{10,1}$ & $\begin{array}{l}\text { Free- } \\
\text { flow }^{3,2}\end{array}$ & Stop-go 7,1 & Free-flow 9,1 & $\begin{array}{l}\text { Free- } \\
\text { flow }^{5,1}\end{array}$ & Free-flow ${ }^{8,1}$ & 1.28 & 5.6 & 225 \\
\hline R13 & $\begin{array}{c}\text { Free-flow } \\
1,1\end{array}$ & $\begin{array}{c}\text { Multiple } \\
\text { stopping2,1 }\end{array}$ & $\begin{array}{l}\text { Stop- } \\
\text { go }^{4,1}\end{array}$ & $\begin{array}{l}\text { Free- } \\
\text { flow }^{6,1}\end{array}$ & Free-flow ${ }^{10,1}$ & $\begin{array}{l}\text { Free- } \\
\text { flow }^{3,1}\end{array}$ & Free-flow ${ }^{7,2}$ & Free-flow ${ }^{9,1}$ & Stop-go ${ }^{5,1}$ & Stop-go ${ }^{8,1}$ & 3.33 & 1.1 & 270 \\
\hline R14 & $\begin{array}{c}\text { Free-flow } \\
1,4\end{array}$ & Stop-go ${ }^{2,1}$ & $\begin{array}{c}\text { Multiple } \\
\text { stopping } \\
, 1\end{array}$ & $\begin{array}{l}\text { Free- } \\
\text { flow }^{6,1}\end{array}$ & Free-flow ${ }^{10,1}$ & $\begin{array}{l}\text { Free- } \\
\text { flow }^{3,1}\end{array}$ & Free-flow ${ }^{7,2}$ & Stop-go ${ }^{9,1}$ & $\begin{array}{l}\text { Free- } \\
\text { flow }^{5,1}\end{array}$ & Stop-go ${ }^{8,1}$ & 3.35 & 0.8 & 270 \\
\hline R15 & $\begin{array}{c}\text { Free-flow } \\
1,4\end{array}$ & Stop-go ${ }^{2,1}$ & $\begin{array}{c}\text { Stop- } \\
\text { go }^{4,1}\end{array}$ & $\begin{array}{l}\text { Stop- } \\
\text { go }^{6,1}\end{array}$ & Free-flow ${ }^{10,1}$ & $\begin{array}{l}\text { Free- } \\
\text { flow }^{3,1}\end{array}$ & Stop-go ${ }^{7,1}$ & Stop-go ${ }^{9,1}$ & $\begin{array}{l}\text { Free- } \\
\text { flow }^{5,1}\end{array}$ & Stop-go ${ }^{8,1}$ & 4.05 & 0.8 & 270 \\
\hline R16 & $\begin{array}{c}\text { Free-flow } \\
1,4\end{array}$ & Free-flow ${ }^{2,1}$ & $\begin{array}{l}\text { Stop- } \\
\text { go }^{4,1}\end{array}$ & $\begin{array}{l}\text { Free- } \\
\text { flow }^{6,1}\end{array}$ & Free-flow ${ }^{10,1}$ & $\begin{array}{l}\text { Free- } \\
\text { flow }^{3,1}\end{array}$ & Stop-go ${ }^{7,1}$ & Stop-go ${ }^{9,1}$ & Stop-go ${ }^{5,1}$ & Stop-go ${ }^{8,1}$ & 5.61 & 0.3 & 270 \\
\hline $\mathrm{R} 17$ & $\begin{array}{c}\text { Free-flow } \\
1,4\end{array}$ & Stop-go ${ }^{2,1}$ & $\begin{array}{l}\text { Stop- } \\
\text { go }^{4,1}\end{array}$ & $\begin{array}{l}\text { Stop- } \\
\text { go }^{6,1}\end{array}$ & Free-flow ${ }^{10,1}$ & $\begin{array}{l}\text { Free- } \\
\text { flow }^{3,1}\end{array}$ & Stop-go ${ }^{7,1}$ & - & Stop-go $0^{5,1}$ & Free-flow 8,1 & 3.64 & 0.3 & 270 \\
\hline R18 & Stop-go ${ }^{1,1}$ & $\begin{array}{c}\text { Multiple } \\
\text { stopping2,1 }\end{array}$ & $\begin{array}{l}\text { Stop- } \\
\text { go }^{4,1}\end{array}$ & $\begin{array}{l}\text { Stop- } \\
\text { go }^{6,1}\end{array}$ & - & $\begin{array}{l}\text { Free- } \\
\text { flow }^{3,1}\end{array}$ & $\begin{array}{c}\text { Multiple } \\
\text { stopping }\end{array}$ & - & $\begin{array}{c}\text { Multiple } \\
\text { stoppings,1 }\end{array}$ & Stop-go ${ }^{8,1}$ & 1.92 & 3.9 & 270 \\
\hline R19 & $\begin{array}{c}\text { Multiple } \\
\text { stopping1,4 }\end{array}$ & Stop-go ${ }^{2,1}$ & $\begin{array}{l}\text { Multiple } \\
\text { stopping }{ }^{4} \\
, 1\end{array}$ & $\begin{array}{l}\text { Stop- } \\
\text { go }^{6,1}\end{array}$ & Stop-go ${ }^{10,1}$ & $\begin{array}{l}\text { Free- } \\
\text { flow }^{3,1}\end{array}$ & $\begin{array}{c}\text { Multiple } \\
\text { stopping }\end{array}$ & Free-flow 9,1 & $\begin{array}{l}\text { Free- } \\
\text { flow }^{5,1}\end{array}$ & Free-flow 8,1 & 2.85 & 3.1 & 225 \\
\hline $\mathrm{R} 20$ & Stop-go ${ }^{1,4}$ & Stop-go ${ }^{2,1}$ & $\begin{array}{l}\text { Multiple } \\
\text { stopping } \\
, 1\end{array}$ & $\begin{array}{c}\text { Multiple } \\
\text { stopping } \\
6,1\end{array}$ & Stop-go ${ }^{10,1}$ & $\begin{array}{l}\text { Free- } \\
\text { flow }^{3,1}\end{array}$ & Stop-go 7,1 & $\begin{array}{c}\text { Multiple } \\
\text { stopping }\end{array}$ & Stop-go ${ }^{5,1}$ & Stop-go ${ }^{8,1}$ & 1.99 & 2.8 & 200 \\
\hline $\mathrm{R} 21$ & Stop-go ${ }^{1,4}$ & Stop-go ${ }^{2,1}$ & $\begin{array}{c}\text { Stop- } \\
\text { go }^{4,1}\end{array}$ & $\begin{array}{l}\text { Free- } \\
\text { flow }^{6,1}\end{array}$ & Free-flow ${ }^{10,1}$ & $\begin{array}{l}\text { Free- } \\
\text { flow }{ }^{3,1}\end{array}$ & Stop-go ${ }^{7,1}$ & Free-flow 9,1 & $\begin{array}{c}\text { Multiple } \\
\text { stoppings,1 }\end{array}$ & Free-flow ${ }^{8,1}$ & 1.96 & 2.8 & 200 \\
\hline $\mathrm{R} 22$ & $\begin{array}{c}\text { Free-flow } \\
1,4\end{array}$ & Stop-go 2,1 & $\begin{array}{l}\text { Stop- } \\
\text { go }^{4,1}\end{array}$ & $\begin{array}{l}\text { Free- } \\
\text { flow }^{6,1}\end{array}$ & Free-flow ${ }^{10,1}$ & $\begin{array}{l}\text { Free- } \\
\text { flow }^{3,1}\end{array}$ & Free-flow ${ }^{7,1}$ & Free-flow ${ }^{9,1}$ & Stop-go 5,1 & Free-flow ${ }^{8,1}$ & 2.16 & 3.3 & 200 \\
\hline
\end{tabular}

"-" refer to data not recorded due to instrumentation error such as communication failure between the instrument and the laptop. First digit of superscript in each cell represents number of a TI and the second digit represents driving direction number. For example, superscript 1, 2 represents $\mathrm{TI}_{1}$ and driving direction 2. Details of driving direction at each of the $\mathrm{TI}$ is shown in Fig. 2.

Cite this article as: Goel, A., Kumar, P., 2015. Zone of influence for particle number concentrations at signalised traffic intersections, Atmospheric Environment 123, 2538. Online Link: http://dx.doi.org/10.1016/j.atmosenv.2015.10.054 
Table 5. Start $\left(X_{1}\right)$ and end points $\left(X_{2}\right)$ of maximum, minimum, median and average distance, represting ZoI for PNCs at four different types of TIs during stop and go driving conditions. Average values for each type of a TI is shown in bold. Detailed runwise information of $X_{1}$ and $X_{2}$ is provided in the SI Table S1.

\begin{tabular}{|c|c|c|c|c|c|c|c|c|}
\hline \multirow{2}{*}{$\begin{array}{l}\text { Type of } \\
\text { TIs }\end{array}$} & \multicolumn{2}{|c|}{ Maximum } & \multicolumn{2}{|c|}{ Minimum } & \multicolumn{2}{|c|}{ Median } & \multicolumn{2}{|c|}{ Average \pm stdev } \\
\hline & $X_{1}(\mathrm{~m})$ & $X_{2}(\mathrm{~m})$ & $X_{1}(\mathrm{~m})$ & $X_{2}(\mathrm{~m})$ & $X_{1}(\mathrm{~m})$ & $X_{2}(\mathrm{~m})$ & $X_{1}(\mathrm{~m})$ & $X_{2}(\mathrm{~m})$ \\
\hline $\mathrm{TI}_{1}$ & 117 & -143 & 16 & -7 & 63 & -42 & $64 \pm 35$ & $-51 \pm 43$ \\
\hline $\mathrm{TI}_{2}$ & 140 & -120 & 27 & 0 & 65 & -45 & $67 \pm 35$ & $-47 \pm 49$ \\
\hline $\mathrm{TI}_{4}$ & 189 & -190 & 0 & -6 & 83 & -17 & $91 \pm 55$ & $-27 \pm 76$ \\
\hline $\mathrm{TI}_{6}$ & 123 & -154 & 40 & 21 & 104 & 21 & $92 \pm 34$ & $-41 \pm 96$ \\
\hline $\mathrm{TI}_{4 \mathrm{w}-\mathrm{nb}}$ & 189 & -190 & $\mathbf{0}$ & -6 & 74 & -30 & $79 \pm 15$ & $-42 \pm 11$ \\
\hline $\mathrm{TI}_{10}$ & 80 & -40 & 10 & -20 & 50 & -40 & $46 \pm 25$ & $-33 \pm 10$ \\
\hline $\mathrm{TI}_{4 \mathrm{w}-\mathrm{wb}}$ & 80 & -40 & 10 & -20 & 50 & -40 & $46 \pm 25$ & $-33 \pm 10$ \\
\hline $\mathrm{TI}_{3}$ & 90 & -50 & 50 & 20 & 70 & 13 & $59 \pm 33$ & $-4 \pm 45$ \\
\hline $\mathrm{TI}_{7}$ & 157 & -111 & 19 & 17 & 89 & -32 & $79 \pm 51$ & $-17 \pm 60$ \\
\hline $\mathrm{TI}_{9}$ & 142 & -98 & 22 & 12 & 68 & -40 & $76 \pm 44$ & $-23 \pm 57$ \\
\hline $\mathbf{T I}_{3 w-n b}$ & 157 & -111 & 19 & 17 & 70 & -32 & $71 \pm 11$ & $-15 \pm 10$ \\
\hline $\mathrm{TI}_{5}$ & 146 & -151 & 65 & 14 & 106 & -98 & $108 \pm 27$ & $-98 \pm 38$ \\
\hline $\mathrm{TI}_{8}$ & 90 & -60 & 40 & 30 & 80 & -30 & $70 \pm 27$ & $-20 \pm 46$ \\
\hline $\mathrm{TI}_{3 \mathrm{w}-\mathrm{wb}}$ & 146 & -151 & 40 & 30 & 93 & -64 & $89 \pm 27$ & $-59 \pm 55$ \\
\hline
\end{tabular}

\subsubsection{ZoI during stop and go driving conditions}

Stop and go driving conditions occur most frequently at the TIs (Table 4). During such conditions, a vehicle undergoes a series of changes in the form of deceleration, idling and acceleration. Interestingly, the maximum length of $\mathrm{ZoI}$ of $\mathrm{TI}_{4 \mathrm{w}-\mathrm{nb}}$ was over 3-fold to those found for the $\mathrm{TI}_{4 \mathrm{w}-\mathrm{wb}}$, but the corresponding difference in average PNCs within the ZoI was only about 23\%. While at three-way TIs, both the length of ZoI at $\mathrm{TI}_{3 \mathrm{w} \text {-wb }}$ and average PNCs within them were $11 \%$ higher than those at $\mathrm{TI}_{3 \mathrm{w}-\mathrm{nb}}$ (Table 7). This can be explained by $\sim 40 \%$ higher driving speed at $\mathrm{T}_{4 \mathrm{w}-\mathrm{nb}}$ than those at $\mathrm{TI}_{4 \mathrm{w} \text {-wb }}$ while average driving speed at both three-way TIs was almost equal (Table 7). Particle number emission increases with the increase in driving speed due to higher engine load (Imhof et al., 2005; Kumar et al., 2011; Wang et al., 2010). The above observations allowed to conclude that irrespective of any type of a TI, maximum length

Cite this article as: Goel, A., Kumar, P., 2015. Zone of influence for particle number concentrations at signalised traffic intersections, Atmospheric Environment 123, 25-38. Online Link: http://dx.doi.org/10.1016/j.atmosenv.2015.10.054 
of ZoI depends predominantly on idling time while average PNCs depends on driving speed of the road vehicles.

Table 6. Start $\left(X_{1}\right)$ and end points $\left(X_{2}\right)$ of maximum, minimum, median and average distance, represting ZoI for PNCs at four different types of TIs during multiple stopping driving conditions. Average values for each type of a TI is shown in bold. Detailed runwise information of $X_{1}$ and $X_{2}$ is provided in the SI Table S2. "-" refers to unavilability of data.

\begin{tabular}{|c|c|c|c|c|c|c|c|c|}
\hline \multirow{2}{*}{$\begin{array}{l}\text { Type of } \\
\text { TIs }\end{array}$} & \multicolumn{2}{|c|}{ Maximum } & \multicolumn{2}{|c|}{ Minimum } & \multicolumn{2}{|c|}{ Median } & \multicolumn{2}{|c|}{ Average \pm stdev } \\
\hline & $X_{1}(\mathrm{~m})$ & $X_{2}(\mathrm{~m})$ & $X_{1}$ & $X_{2}(\mathrm{~m})$ & $X_{1}$ & $X_{2}(\mathrm{~m})$ & $X_{1}(\mathrm{~m})$ & $X_{2}(\mathrm{~m})$ \\
\hline $\mathrm{TI}_{1}$ & 120 & -187 & 20 & -178 & 70 & -183 & $70 \pm 70$ & $-182 \pm 6$ \\
\hline $\mathrm{TI}_{2}$ & 205 & -21 & 98 & 10 & 147 & 13 & $149 \pm 37$ & $5 \pm 16$ \\
\hline $\mathrm{TI}_{4}$ & 138 & -91 & 58 & 28 & 74 & -35 & $89 \pm 32$ & $-23 \pm 53$ \\
\hline $\mathrm{TI}_{6}$ & 158 & 58 & 158 & 58 & 158 & 58 & - & - \\
\hline $\mathbf{T I}_{4 w-n b}$ & 205 & -190 & $\mathbf{0}$ & -6 & 134 & -11 & $119 \pm 30$ & $\begin{array}{c}- \\
68 \pm 104\end{array}$ \\
\hline $\mathrm{TI}_{10}$ & - & - & - & - & - & - & - & - \\
\hline $\mathrm{TI}_{4 \mathrm{w}-\mathrm{wb}}$ & - & - & - & - & - & - & - & - \\
\hline $\mathrm{TI}_{3}$ & - & - & - & - & - & - & - & - \\
\hline $\mathrm{TI}_{7}$ & 169 & 40 & 31 & 5 & 71 & 19 & $76 \pm 48$ & $20 \pm 10.5$ \\
\hline $\mathrm{TI}_{9}$ & 196 & 57 & 62 & 39 & 107 & -1 & $118 \pm 65$ & $-5 \pm 41$ \\
\hline TI $I_{3 w-n b}$ & 196 & 40 & 31 & 5 & 89 & 9 & $97 \pm 30$ & $8 \pm 17$ \\
\hline $\mathrm{TI}_{5}$ & 160 & -96 & 111 & 28 & 130 & -79 & $134 \pm 25$ & $-45 \pm 74$ \\
\hline $\mathrm{TI}_{8}$ & - & - & - & - & - & - & - & - \\
\hline $\mathrm{TI}_{3 \mathrm{w} \text {-wb }}$ & 160 & -96 & 111 & 28 & 130 & -79 & $134 \pm 25$ & $-45 \pm 74$ \\
\hline
\end{tabular}

The median length of ZoI at $\mathrm{TI}_{4 \mathrm{w} \text {-nb }}$ was $15 \%$ higher than that of $\mathrm{TI}_{4 \mathrm{w}-\mathrm{wb}}$ and the corresponding difference in average PNCs was only 25\%. While at TI $3 \mathrm{w}$-wb, length of ZoI was $\sim 54 \%$ more than those at $\mathrm{TI}_{3 \mathrm{w}-\mathrm{nb}}$ and corresponding differences in PNCs were only about 3\% (Table 7). Similar to average PNCs in maximum length of ZoI, a linear correlation $\left(R^{2}=0.49\right)$ was found between average PNCs in median length and the driving speed of the traffic. These observations suggested that for all types of TIs, median length of ZoI depends predominantly on the acceleration of traffic.

Above discussions clearly show that ZoI exists within the vicinity of a TI and the length of a ZoI is dissimilar at different types of TIs. For a driving condition, ZoI and PNCs

Cite this article as: Goel, A., Kumar, P., 2015. Zone of influence for particle number concentrations at signalised traffic intersections, Atmospheric Environment 123, 25-38. Online Link: http://dx.doi.org/10.1016/j.atmosenv.2015.10.054 
within them depends on factors such as average acceleration, deceleration and idling time (Table 7).

Table 7. Average deceleration, acceleration, driving speed, idling time and PNCs within maximum and median lengths of ZoI at four different types of TIs in stop and go driving conditions.

\begin{tabular}{|c|c|c|c|c|c|c|}
\hline $\begin{array}{l}\text { Type of } \\
\text { TIs }\end{array}$ & ZoI (m) & $\begin{array}{l}\text { Deceleration } \\
\left(\mathrm{m} \mathrm{s}^{-2}\right)\end{array}$ & $\begin{array}{l}\text { Acceleration } \\
\left(\mathrm{m} \mathrm{s}^{-2}\right)\end{array}$ & $\begin{array}{l}\text { Driving } \\
\text { speed } \\
\left(\mathrm{km} \mathrm{h}^{-1}\right)\end{array}$ & $\begin{array}{l}\text { Idling } \\
\text { time } \\
\text { (s) }\end{array}$ & $\begin{array}{l}\text { PNC } \\
\left(\# \mathrm{~cm}^{-3}\right) \\
\times 10^{5}\end{array}$ \\
\hline \multicolumn{7}{|c|}{ Maximum length } \\
\hline $\mathrm{TI}_{4 \mathrm{w}-\mathrm{nb}}$ & 379 & -0.61 & 0.68 & 19 & 47 & 1.09 \\
\hline $\mathrm{TI}_{4 \mathrm{w}-\mathrm{wb}}$ & 120 & -0.87 & 0.83 & 13 & 15 & 0.89 \\
\hline $\mathrm{TI}_{3 \mathrm{w}-\mathrm{nb}}$ & 268 & -0.74 & 0.92 & 17 & 25 & 0.97 \\
\hline $\mathrm{TI}_{3 \mathrm{w}-\mathrm{wb}}$ & 297 & -0.77 & 0.96 & 18 & 23 & 1.08 \\
\hline \multicolumn{7}{|c|}{ Median length } \\
\hline $\mathrm{TI}_{4 \mathrm{w}-\mathrm{nb}}$ & 104 & -0.48 & 0.70 & 20 & 47 & 1.22 \\
\hline $\mathrm{TI}_{4 \mathrm{w}-\mathrm{wb}}$ & 90 & -0.67 & 0.74 & 15 & 15 & 0.97 \\
\hline $\mathrm{TI}_{3 \mathrm{w}-\mathrm{nb}}$ & 102 & -0.57 & 0.86 & 14 & 25 & 1.08 \\
\hline $\mathrm{TI}_{3 \mathrm{w}-\mathrm{wb}}$ & 157 & -0.84 & 0.92 & 15 & 23 & 1.10 \\
\hline
\end{tabular}

\subsubsection{ZoI during multiple stopping driving conditions}

Multiple stopping is a driving condition where a vehicle stops at a TI more than once in a signal cycle due to oversaturation (having traffic volume greater than the designed capacity) of a TI. On our study route, such conditions were encountered at a number occasions at the TIs (see Table 4). The maximum length of $\mathrm{ZoI}$ at $\mathrm{TI}_{3 \mathrm{w} \text {-wb }}$ was

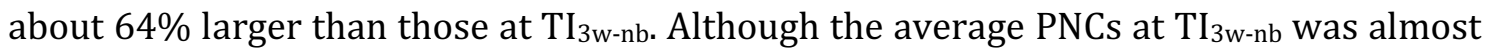
twice to those found at the $\mathrm{TI}_{3 \mathrm{w} \text {-wb }}$ (Table 8). This can be explained by higher acceleration at $\mathrm{TI}_{3 \mathrm{w}-\mathrm{nb}}\left(1.0 \mathrm{~m} \mathrm{~s}^{-2}\right)$ compared with relatively lower acceleration $\left(0.8 \mathrm{~m} \mathrm{~s}^{-}\right.$

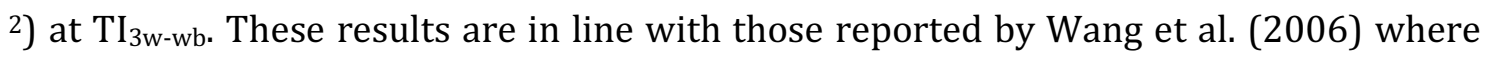
they found that high PNCs were mainly associated with heavy acceleration, high engine speed and high engine torque. These observations allow to conclude that maximum Cite this article as: Goel, A., Kumar, P., 2015. Zone of influence for particle number concentrations at signalised traffic intersections, Atmospheric Environment 123, 25-38. Online Link: http://dx.doi.org/10.1016/j.atmosenv.2015.10.054 
length of ZoI depends on idling time at a TI and average PNCs within ZoI on the acceleration.

Highest average PNCs within median length of ZoI was found at $\mathrm{TI}_{3 \mathrm{w}-\mathrm{nb}}$, followed by $\mathrm{TI}_{4 \mathrm{w}}$

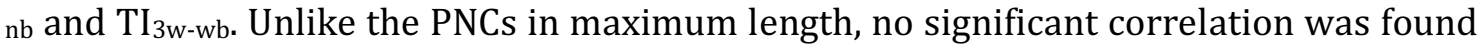
between average PNCs, and studied factors (i.e., acceleration, deceleration, driving speed and idling time) at a TI.

Table 8. Maximum and median length of ZoI, alongwith average deceleration, acceleration, driving speed, idling time and PNCs within them, at four different types of TIs. "-" refers to unavilabity of data.

\begin{tabular}{|c|c|c|c|c|c|c|}
\hline \multirow[b]{2}{*}{ Type of TIs } & \multirow[b]{2}{*}{ ZoI (m) } & \multirow[b]{2}{*}{$\begin{array}{l}\text { Deceleration } \\
\quad\left(\mathrm{m} \mathrm{s}^{-2}\right)\end{array}$} & \multirow[b]{2}{*}{$\begin{array}{l}\text { Acceleration } \\
\left(\mathrm{m} \mathrm{s}^{-2}\right)\end{array}$} & \multirow{2}{*}{$\begin{array}{c}\text { Driving } \\
\text { speed } \\
\left(\mathrm{km} \mathrm{h}^{-}\right. \\
1 \text { ) }\end{array}$} & \multirow[b]{2}{*}{$\begin{array}{l}\text { Idling } \\
\text { time (s) }\end{array}$} & \multirow{2}{*}{$\begin{array}{c}\text { PNC } \\
\left(\# \mathrm{~cm}^{-}\right. \\
3) \times 10^{4}\end{array}$} \\
\hline & & & & & & \\
\hline \multicolumn{7}{|c|}{ Maximum length } \\
\hline $\mathrm{TI}_{4 \mathrm{w}-\mathrm{nb}}$ & 395 & -0.46 & 0.49 & 9 & 97 & 4.87 \\
\hline $\mathrm{TI}_{4 \mathrm{w}-\mathrm{wb}}$ & - & - & - & - & - & - \\
\hline $\mathrm{TI}_{3 \mathrm{w}-\mathrm{nb}}$ & 156 & -0.83 & 1.01 & 12 & 38 & 8.45 \\
\hline $\mathrm{TI}_{3 \mathrm{w}-\mathrm{wb}}$ & 256 & -0.77 & 0.79 & 13 & 23 & 4.14 \\
\hline \multicolumn{7}{|c|}{ Median length } \\
\hline $\mathrm{TI}_{4 \mathrm{w}-\mathrm{nb}}$ & 122 & -0.39 & 0.44 & 6 & 97 & 5.06 \\
\hline $\mathrm{TI}_{4 \mathrm{w}-\mathrm{wb}}$ & - & - & - & - & - & - \\
\hline $\mathrm{TI}_{3 \mathrm{w}-\mathrm{nb}}$ & 80 & -0.73 & 0.81 & 9 & 38 & 9.81 \\
\hline $\mathrm{TI}_{3 \mathrm{w}-\mathrm{wb}}$ & 209 & -0.70 & 0.81 & 13 & 23 & 4.15 \\
\hline
\end{tabular}

\subsection{ZoI during free-flow driving conditions}

Free-flow is a driving condition in which a vehicle crosses a TI without appreciable change in its driving speed. This type of a driving condition occurs less frequently as compare to stop- and go-driving condition at TIs (Table 3). On our study route, this driving condition occurred a few times at the TIs (Table 3). However, there was no juncture $\left(X_{1}\right.$ and $\left.X_{2}\right)$ found between the PNC and driving speed profiles with respect to changing distance from the centre of a TI (Figure 3). An example of average PNC and speed profiles with respect to distance from the centre of a TI at four different Cite this article as: Goel, A., Kumar, P., 2015. Zone of influence for particle number concentrations at signalised traffic intersections, Atmospheric Environment 123, 25-38. Online Link: http://dx.doi.org/10.1016/j.atmosenv.2015.10.054 
types of TIs, which were $\mathrm{TI}_{1}\left(\mathrm{TI}_{4 \mathrm{w}-\mathrm{nb}}\right), \mathrm{TI}_{10}\left(\mathrm{TI}_{4 \mathrm{w}-\mathrm{wb}}\right), \mathrm{TI}_{3}\left(\mathrm{TI}_{3 \mathrm{w}-\mathrm{nb}}\right)$ and $\mathrm{TI}_{8}\left(\mathrm{TI}_{3 \mathrm{w}-\mathrm{wb}}\right)$, is shown in Figure 4. These results clearly suggest that the ZoI does not exist for free flow driving conditions, substantiating previous findings that free-flow traffic conditions at the TIs can eliminate the pollution hotspots around TIs (Fruin et al., 2008; Goel and Kumar, 2015; Kim et al., 2014).
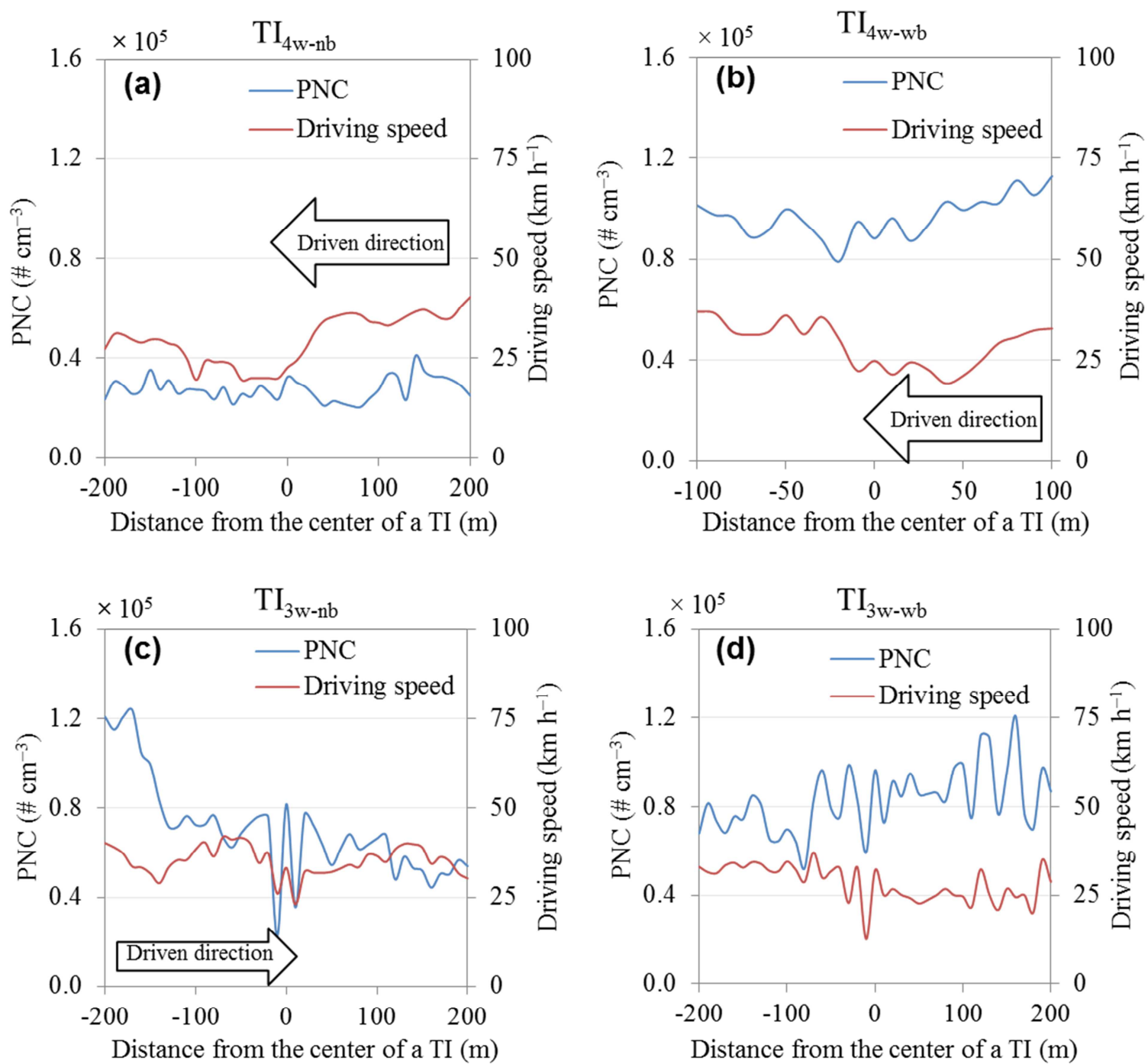

Figure. 4. Distance versus driving speed and PNCs profile in free-flow traffic driving condition at (a) $\mathrm{TI}_{4 \mathrm{w}-\mathrm{nb}}$, (b) $\mathrm{TI}_{4 \mathrm{w}-\mathrm{wb}}$ (c) $\mathrm{TI}_{3 \mathrm{w}-\mathrm{nb}}$ and (d) $\mathrm{TI}_{3 \mathrm{w}-\mathrm{wb}}$. These profiles are plotted

Cite this article as: Goel, A., Kumar, P., 2015. Zone of influence for particle number concentrations at signalised traffic intersections, Atmospheric Environment 123, 25-38. Online Link: http://dx.doi.org/10.1016/j.atmosenv.2015.10.054 
by assuming centre of a TI at zero. Driving direction of an experimental car is shown by an arrow.

\subsection{PNC profiles within ZoI at different types of TIs}

Larger variations in PNCs were observed within the ZoI around the TIs compared with those observed at the remaining length of the route (Section 3.1). We tried different best fitting curves on PNCs within the ZoI. Polynomial form represented the data points well at all the four different types of TIs (SI Fig. S1). A generic form of these polynomial fits is seen in Eq. (1).

$(\mathrm{N})_{\mathrm{x}}\left(\# \mathrm{~cm}^{-3}\right)=\mathrm{a}\left(\# \mathrm{~cm}^{-6}\right) \mathrm{x}^{3}\left(\mathrm{~cm}^{3}\right)+\mathrm{b}\left(\# \mathrm{~cm}^{-5}\right) \mathrm{x}^{2}\left(\mathrm{~cm}^{2}\right)+\mathrm{c}\left(\# \mathrm{~cm}^{-4}\right) \mathrm{x}(\mathrm{cm})+\mathrm{d}\left(\# \mathrm{~cm}^{-3}\right)$

Where $(N)_{x}$ is PNC at a distance ' $x$ ' from the centre of a TI within a ZoI, $x$ can be a curved line following the road; $a, b, c$ and $d$ are coefficients of the polynomial equation. The coefficient $d$ turns out to be equal to the $70^{\text {th }}$ percentile of PNCs within the ZoI. Table 9 shows the values of coefficients $a, b, c$ and $d$, which were derived for all the four different types of $\mathrm{TIs}$ (i.e. $\mathrm{TI}_{2}, \mathrm{TI}_{10}, \mathrm{TI}_{3}$ and $\mathrm{TI}_{8}$ that represent $\mathrm{TI}_{4 \mathrm{w}-\mathrm{nb}}, \mathrm{TI}_{4 \mathrm{w} \text {-wb}}, \mathrm{TI}_{3 \mathrm{w} \text {-nb }}$ and $\mathrm{TI}_{3 \mathrm{w}-\mathrm{wb}}$, respectively).

PNC profiles within the ZoI can depend on numerous factors. These include particle number flux (PNF, i.e. the net number of particles passing through a unit surface area in unit time; Kumar et al., 2008), wind speed (u), average driving speed ( $v$ ), resultant wind speed $(w)$ that is a square root of sum of squares of wind speed and driving speed (i.e. $\sqrt[2]{\alpha u^{2}+\beta v^{2}}$ ), height $(h)$ of buildings around a TI, and the delay time $(t)$ at a TI. Dimensional analysis was performed in order to physically interpret the coefficients of the Eq. (1). Details of the dimensional analysis are presented in SI Section S1 and the final form of it is seen in Eq. (2).

$$
\begin{aligned}
& \begin{array}{ll}
a^{\prime} & b^{\prime} \\
\hline
\end{array} \\
& (\mathrm{N})_{\mathrm{x}}-\mathrm{N}_{70 \text { th }}=(P N F) \times\left(\frac{1}{w}\right) \times\left(\frac{1}{h}\right)\left\{\left(\frac{1}{u}\right) \times\left(\frac{1}{v}\right) \times \mathrm{t}^{-2} \mathrm{x}^{3}+\left(\frac{1}{u}\right) \times \mathrm{t}^{-1} \mathrm{x}^{2}+\mathrm{x}\right\}
\end{aligned}
$$

Based on the dimensional equality, the dimension of $a, b$ and $c$ should be $\# \mathrm{~cm}^{-6}, \# \mathrm{~cm}^{-5}$ and $\# \mathrm{~cm}^{-4}$. The maximum dimension that is common among $a, b$ and $c$ is $\# \mathrm{~cm}^{-4}$.

Cite this article as: Goel, A., Kumar, P., 2015. Zone of influence for particle number concentrations at signalised traffic intersections, Atmospheric Environment 123, 25-38. Online Link: http://dx.doi.org/10.1016/j.atmosenv.2015.10.054 
Therefore, coefficient $a$ and $b$ are normalised against coefficient c and normalised coefficient are represented as $a^{\prime}$ and $b^{\prime}$, respectively.

The coefficient $c$, which was found to be directly proportional to PNF (see Eq. 2 and SI Section S1), represents the influence of PNF on PNCs profiles. The value of $c$ was found to be higher at $\mathrm{TI}_{4 \mathrm{w}-\mathrm{nb}}$ than those at $\mathrm{TI}_{4 \mathrm{w} \text {-wb }}$ (Table 9). Since PNF is directly proportional to traffic volume, which was $\sim 2$ times higher at $\mathrm{TI}_{4 \mathrm{w}-\mathrm{nb}}$ than those at $\mathrm{TI}_{4 \mathrm{w}-\mathrm{wb}}$, led to

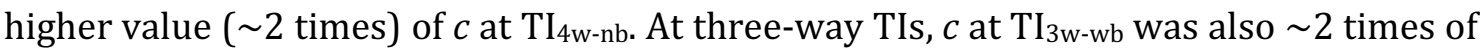
$\mathrm{TI}_{3 \mathrm{w}-\mathrm{nb}}$. This can be explained by higher traffic volume at $\mathrm{TI}_{3 \mathrm{w}-\mathrm{wb}}$ as compare to $\mathrm{TI}_{3 \mathrm{w} \text {-nb }}$. The above results indicate that $c$ is more influenced by traffic volume as compare to other parameters at both four-way and three-way TIs.

Table 9. Values of coefficients ' $a$ ', ' $b$ ', ' $c$ ' and ' $N_{70 t h}$ ' at two different types of four-way TIs

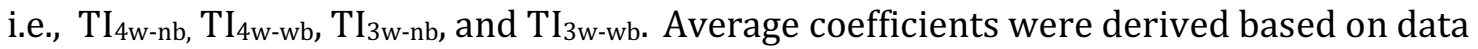
of nine, nine, five and three runs in case of $\mathrm{TI}_{4 \mathrm{w}-\mathrm{nb}}, \mathrm{TI}_{4 \mathrm{w}-\mathrm{wb}}, \mathrm{TI}_{3 \mathrm{w}-\mathrm{nb}}$, and $\mathrm{TI}_{3 \mathrm{w}-\mathrm{wb}}$, respectively.

\begin{tabular}{lllll}
\hline Type of a TI & $a\left(\# \mathrm{~cm}^{-6}\right)$ & $b\left(\# \mathrm{~cm}^{-5}\right)$ & $c\left(\# \mathrm{~cm}^{-4}\right)$ & $d\left(\# \mathrm{~cm}^{-3}\right)$ \\
\hline $\mathrm{TI}_{4 \mathrm{w}-\mathrm{nb}}$ & -0.45 & -6.45 & 1218 & 244537 \\
\hline $\mathrm{TI}_{4 \mathrm{w}-\mathrm{wb}}$ & -0.41 & -12.51 & 952 & 126833 \\
\hline $\mathrm{TI}_{3 \mathrm{w}-\mathrm{nb}}$ & -0.18 & 23.79 & -272 & 42709 \\
\hline $\mathrm{TI}_{3 \mathrm{w}-\mathrm{wb}}$ & 0.44 & -54.12 & -472 & 248415 \\
\hline
\end{tabular}

Normalised coefficient $a^{\prime}$ represents the combined influence of wind and driving speed along with delay time on PNCs profiles. The values of $a^{\prime}$ was higher at TIs with built-up areas as compare to TIs without built-up area. For instance, $a^{\prime}\left(9.3 \times 10^{-4} \mathrm{~m}^{-2}\right)$ was 2.5 times of $\mathrm{TI}_{4 \mathrm{w}-\mathrm{nb}}$ at $\mathrm{TI}_{4 \mathrm{w} \text {-wb}}$, and 1.5-times of $\mathrm{TI}_{3 \mathrm{w} \text {-nb }}$ at $\mathrm{TI}_{3 \mathrm{w} \text {-wb }}$ (Table 9). These differences can be explained by changes in $u, v$ and $t$ at different TIs since these factors are inversely proportional $a^{\prime}$ (see Eq. 2 and SI Section S1). The delay time, $t$, at TI ${ }_{4 \mathrm{w}-\mathrm{nb}}$ was $\sim 3$-fold

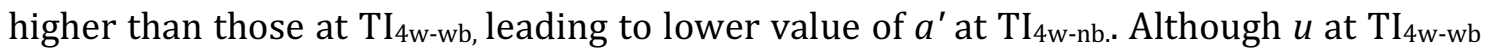
was $\sim 1.7$ times of $\mathrm{TI}_{4 \mathrm{w}-\mathrm{nb}}$ and $v$ of vehicle was almost same at both TIs, which shows that $a^{\prime}$ is more influenced by $t$ at a four-way TIs as compare to $u$ and $v$. At three-way TIs, $t$

Cite this article as: Goel, A., Kumar, P., 2015. Zone of influence for particle number concentrations at signalised traffic intersections, Atmospheric Environment 123, 25-38. Online Link: http://dx.doi.org/10.1016/j.atmosenv.2015.10.054 
and $v$ were almost similar at both $\mathrm{TI}_{3 \mathrm{w} \text {-wb }}$ and $\mathrm{TI}_{3 \mathrm{w} \text {-nb }}$ but $u$ at $\mathrm{TI}_{3 \mathrm{w} \text {-nb }}$ was $\sim 2$ times of

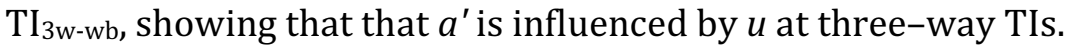

Unlike a', b' shows the dependency of PNC profiles only on wind speed and delay time. As seen in the Table 9, $b^{\prime}$ at $\mathrm{TI}_{4 \mathrm{w} \text {-wb }}\left(-1.31 \times 10^{-2} \mathrm{~m}^{-2}\right)$ and $\mathrm{TI}_{3 \mathrm{w} \text {-wb }}\left(1.15 \times 10^{-1} \mathrm{~m}^{-2}\right)$ was about 2.5- and 1.3-times to those found at $\mathrm{TI}_{4 \mathrm{w}-\mathrm{nb}}$ and $\mathrm{TI}_{3 \mathrm{w}-\mathrm{nb}}$, respectively. These differences can be explained by the changes in $u$ and $t$ at different TIs since these factors are inversely proportional to $b^{\prime}$ (see Eq. 2 and SI Section S1). The $t$ at $\mathrm{TI}_{4 \mathrm{w}-\mathrm{nb}}$ was about 1.2-times higher than those at $\mathrm{TI}_{4 \mathrm{w} \text {-wb }}$, leading to lower value of $b^{\prime}$ at $\mathrm{TI}_{4 \mathrm{w} \text {-nb }}$ while $u$ was almost same at both TIs. In case of three-way TIs, $t$ was almost same at both the TIs

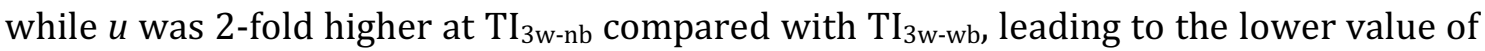

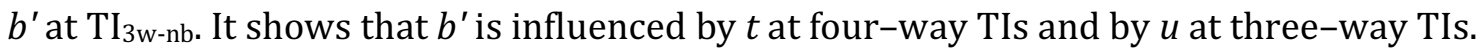

Above discussion showed that PNCs follow a three degree polynomial profile within ZoI of a TI. Less variability among the values of coefficients was observed at four-way TIs compared with three-way TIs. At four-way TIs, coefficients were mainly influenced by delay time and traffic volume while wind speed and traffic volume played an important role in determining coefficients at the three-way TIs.

\subsection{PMF modelling}

Receptor models such as PMF are mostly applied to ambient particle chemical composition data in such a way that solution is combination of certain number of emission sources that are known as factors. By applying PMF to on-road PND data, results obtained were driving conditions (instead of emission sources), and the size distribution of emissions in such driving conditions (instead of chemical profiles) (Domínguez-Sáez et al., 2012). This approach of PMF analysis is applied separately on the PND data for the whole route as well as those within the ZoI of four different types of TIs (Figure. 5).

The model was run separately by considering solutions for 2, 3, 4 and 5 factors. A 4factor solution was considered as optimal because: (a) theoretical value of Q (692294), which is a goodness of a fit parameter of chi square distribution (JRC, 2009) and determines how well the model fit the input data, was found to be approximately equal to the number of data points in the array (784754), and (b) the factors identified were Cite this article as: Goel, A., Kumar, P., 2015. Zone of influence for particle number concentrations at signalised traffic intersections, Atmospheric Environment 123, 25-38. Online Link: http://dx.doi.org/10.1016/j.atmosenv.2015.10.054 
found to be related to known physical sources (Domínguez-Sáez et al., 2012; Gupta et al., 2012; Hopke, 2000). Identified factors were tentatively assigned to the potential sources based on the following information: (i) comparison of factor specific PNDs profile reported in this study with those available in literature (Figure 5a-d; Table 10), (ii) comparison of average of normalised factor contributions for different traffic speed and acceleration combinations (Figure 5e), and (iii) normalised factor contribution and driving speed profiles with respect to distance from the centre of a TI at four different types of TIs (Figures 5j, o, $\mathrm{t}$ and $\mathrm{y}$ ). Robustness of the results was further confirmed based on similarity in factor specific PND profiles for the whole route as well as at four different types of TIs (Figure 5f-x). After factor identification, the contribution of each factor to total PNCs was calculated using multiple linear regressions (Domínguez-Sáez et al., 2012).

\subsubsection{Allocation of factors to different driving conditions}

Factor specific PND profiles were found to be identical in shape for the different types of TIs and the whole route (Figure 5). The results related to $\mathrm{TI}_{4 \mathrm{w}-\mathrm{nb}}$ are therefore discussed in detail below. Detailed discussion on contribution of PNCs released due to each of these factors to total PNCs is provided in Section 3.4.2.

Factor 1 (Deceleration): Factor 1 showed a bimodal PND profile with peaks at diameter $\sim 8$ and $100 \mathrm{~nm}$ (Figure 5f). Similar bimodal distribution with peak at diameter about 10 and $100 \mathrm{~nm}$ was observed by Wang et al. (2006) during deceleration of diesel vehicles (Table 10). Similarly, Huang et al. (2012) and Karajalainen et al. (2014) observed PNDs mainly composed of nucleation mode particles during deceleration driving conditions (Table 10). Our PND profiles were also found to be dominated by nucleation mode particles of less than $20 \mathrm{~nm}$ in diameter and therefore attributed to deceleration conditions (Table 10). During deceleration, fuel supply to the engine is reduced due to which emissions of soot particles drop, which reduces the available surface area for condensation of organic material, and sulphuric acid and thus nucleation occurs (Vogt et al., 2003). Further average normalised contribution of factor 1 was highest during deceleration (see Figure 5e). Normalised contribution of factor 1 with respect to distance at TIs increases with decrease in driving speed of the

Cite this article as: Goel, A., Kumar, P., 2015. Zone of influence for particle number concentrations at signalised traffic intersections, Atmospheric Environment 123, 25-38. Online Link: http://dx.doi.org/10.1016/j.atmosenv.2015.10.054 
experimental car at each of the four types of TIs (see Figure 5j, o, t and y). Therefore based on above discussions, factor 1 was identified as deceleartion.

Factor 2 (Aceleration): Factor 2 showed a bimodal factor specific PND profile with first peak at diameter $\sim 30 \mathrm{~nm}$ and second peak diameter varied from 205-400 nm (Figure 5g). Similar bimodal PND with peaks at $\sim 20$ and 70-150 $\mathrm{nm}$ during acceleration was reported by Shah and Cocker (2005), Karjalainen et al. (2014) and Goel and Kumar (2015). During acceleration, PNCs are typically higher due to higher engine loading that leads to greater fuel consumption (Karjalainen et al., 2014). Further normalised contribution of factor 2 was higher compared with other factors (i.e., factor 3 and factor 4) during acceleration (Figure 5e). Normalised contribution of factor 2 with respect to distance at TIs showed an increasing trend with increase in the driving speed of the experimental car at each of the four types of TIs (Figure $5 \mathrm{j}, \mathrm{o}, \mathrm{t}$ and $\mathrm{y}$ ). Based on above discussion, this factor is identified as acceleration.Factor 3 (Cruising): Factor 3 showed a trimodal factor specific PND profile with peaks at approximately 5, 25 and $100 \mathrm{~nm}$ (Figure 5h). Similar trimodal PND with peaks at 5, 20 and $100 \mathrm{~nm}$ during cruising was reported by Shah and Cocker (2005). Further, relative average normalised contribution of factor 3 was higher during cruising driving condition as compare to factor 4 (see Figure 5e). Therefore, this factor is identified as cruising.

Factor 4 (Creep-idling): Factor 4 showed a trimodal factor specific PND profile with peaks at 5, 48 and $100 \mathrm{~nm}$ (Figure 5i). Similar trimodal PNDs with peaks at 5, 40 and $100 \mathrm{~nm}$ during idling of diesel bus in a road chasing experiment in Korea was observed by Kwak et al. (2014). Further the contribution of this factor was highest during creepidling (Figure 5e) and therefore this factor is identified as creep-idling.

Cite this article as: Goel, A., Kumar, P., 2015. Zone of influence for particle number concentrations at signalised traffic intersections, Atmospheric Environment 123, 25-38. Online Link: http://dx.doi.org/10.1016/j.atmosenv.2015.10.054 


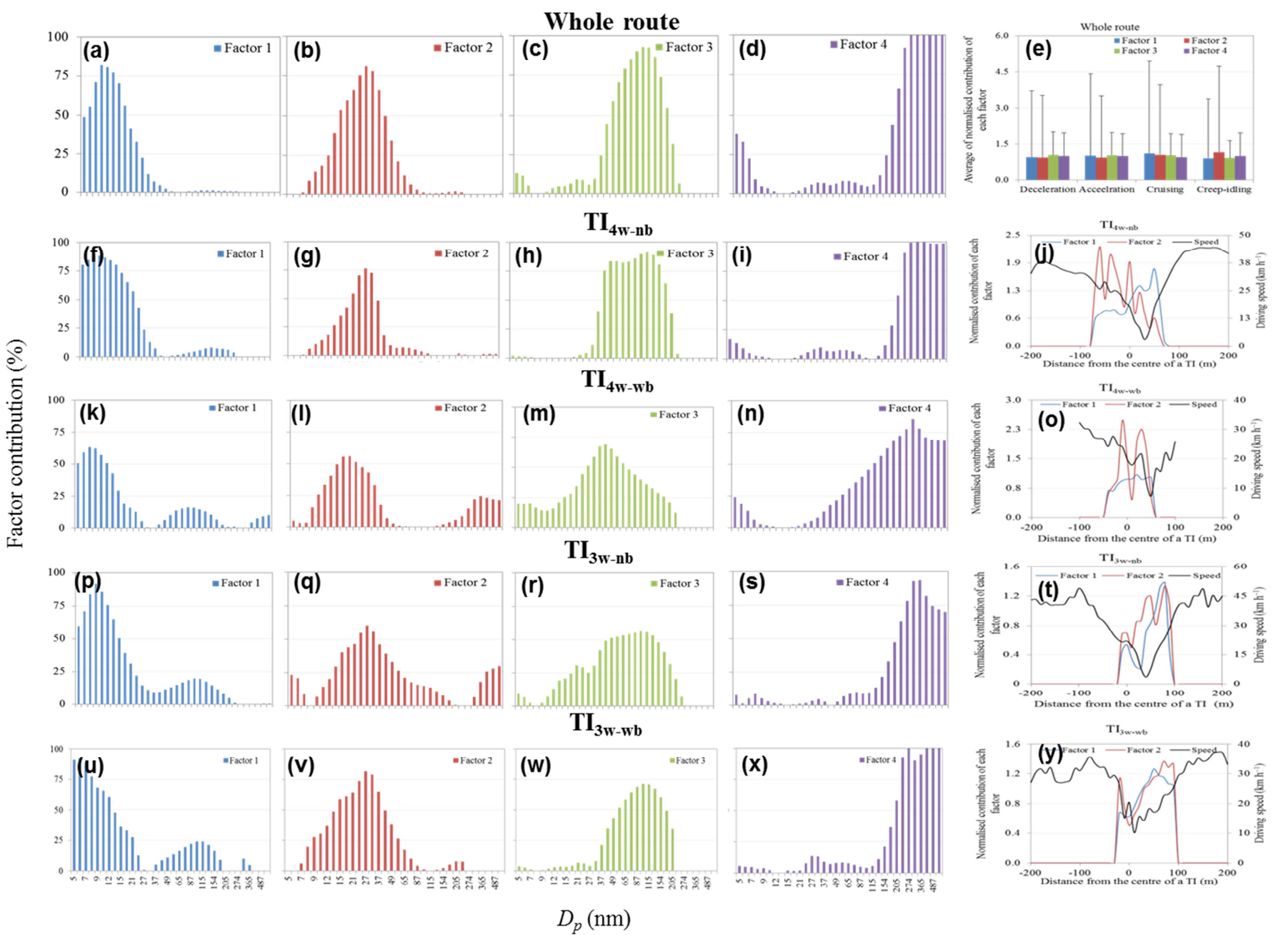

Figure. 5. Percentage contribution of each factor identified towards the PNCs in different size ranges on whole route and at four different types of TIs. Along with average of normalised contribution of factor 1 , factor 2 , factor 3 and factor 4 during deceleration, acceleration, cruising and creep-idling on the whole route. Also, profile of normalised contribution of factor 1 and factor 2 with respect to distance from the centre of a TI at four different types of TIs are presented.

Cite this article as: Goel, A., Kumar, P., 2015. Zone of influence for particle number concentrations at signalised traffic intersections, Atmospheric Environment 123, 2538. Online Link: http://dx.doi.org/10.1016/j.atmosenv.2015.10.054 
Table 10. Summary of field studies discussing PNDs characteristics during different driving conditions.

\begin{tabular}{|c|c|c|c|c|c|c|}
\hline Study & Type & $\begin{array}{l}\text { Traffic } \\
\text { composition }\end{array}$ & $\begin{array}{l}\text { Range } \\
(\mathrm{nm})\end{array}$ & $\begin{array}{l}\text { Instrumen } \\
\mathrm{t}\end{array}$ & $\begin{array}{l}\text { PNDs } \\
\text { characteristics } \\
\text { in different } \\
\text { driving } \\
\text { conditions }\end{array}$ & $\begin{array}{l}\text { Contribution } \\
\text { of PNCs } \\
\text { released } \\
\text { during } \\
\text { different } \\
\text { driving } \\
\text { conditions }\end{array}$ \\
\hline $\begin{array}{l}\text { Shah and } \\
\text { Cocker } \\
(2005)\end{array}$ & $\begin{array}{l}\text { Engine } \\
\text { exhaust } \\
\text { measurem } \\
\text { ents }\end{array}$ & $\begin{array}{l}\text { Heavy duty } \\
\text { diesel truck }\end{array}$ & $\begin{array}{l}0- \\
1000\end{array}$ & $\begin{array}{l}\text { SMPS, CPC } \\
\text { and DMA }\end{array}$ & $\begin{array}{l}\text { Found } \\
\text { presence of a } \\
\text { number peak } \\
\text { at } 15 \mathrm{~nm} \\
\text { daring } \\
\text { transient } \\
\text { vehicle } \\
\text { operation }\end{array}$ & $\begin{array}{l}\text { They } \\
\text { observed } \\
\text { high PNCs } \\
\text { during } \\
\text { acceleration } \\
\text { and uphill } \\
\text { driving }\end{array}$ \\
\hline $\begin{array}{l}\text { Wang et al. } \\
\text { (2006) }\end{array}$ & $\begin{array}{l}\text { Engine } \\
\text { exhaust } \\
\text { measurem } \\
\text { ents using } \\
\text { heavy duty } \\
\text { transient } \\
\text { test cycle } \\
\text { (HDTT) on } \\
\text { Chassis } \\
\text { dynamome } \\
\text { ter }\end{array}$ & $\begin{array}{l}\text { Heavy duty } \\
\text { diesel pickup } \\
\text { truck }\end{array}$ & $\begin{array}{l}5.6- \\
560\end{array}$ & EEPS & $\begin{array}{l}\text { Acceleration } \\
\text { was dominated } \\
\text { by Aitken } \\
\text { mode particles } \\
\text { with diameter } \\
\text { around } 50 \mathrm{~nm} \text {, } \\
\text { deceleration } \\
\text { was dominated } \\
\text { by nucleation } \\
\text { mode particle } \\
\text { and idling was } \\
\text { dominated by } \\
\text { Aitken mode } \\
\text { with diameter } \\
\text { around } 60 \mathrm{~nm}\end{array}$ & $\begin{array}{l}\text { They found } \\
\text { highest PNCs } \\
\text { during } \\
\text { acceleration } \\
\text { followed by } \\
\text { cruising, } \\
\text { deceleration } \\
\text { and idling }\end{array}$ \\
\hline $\begin{array}{l}\text { Domínguez- } \\
\text { Sáez et al. } \\
\text { (2012) }\end{array}$ & $\begin{array}{l}\text { Positive } \\
\text { matrix } \\
\text { factorizatio } \\
n \text { of } \\
\text { exhaust } \\
\text { sampling } \\
\text { of a diesel } \\
\text { car }\end{array}$ & Diesel car & $\begin{array}{l}12.4- \\
560\end{array}$ & EEPS 3090 & $\begin{array}{l}\text { In cruise, they } \\
\text { found high } \\
\text { PNCs in Aitken } \\
\text { mode particles } \\
\text { (diameter > } \\
29.4 \text { nm), with } \\
\text { a statistical } \\
\text { mode of the } \\
\text { size } \\
\text { distribution at } \\
60 \text { nm. In } \\
\text { transient } \\
\text { conditions } \\
\text { (medium-high } \\
\text { velocity and } \\
\text { high velocity } \\
\text { standard } \\
\text { deviations } \\
\text { with frequent } \\
\text { gear shift), } \\
\text { they found } \\
\text { high PNCs of }\end{array}$ & $\begin{array}{l}\text { Cruise, } \\
\text { transient } \\
\text { driving } \\
\text { conditions } \\
\text { and creep- } \\
\text { idling } \\
\text { contributed } \\
60 \%, 25 \% \\
\text { and } 14 \% \text {, } \\
\text { respectively. }\end{array}$ \\
\hline
\end{tabular}

Cite this article as: Goel, A., Kumar, P., 2015. Zone of influence for particle number concentrations at signalised traffic intersections, Atmospheric Environment 123, 25-38. Online Link: http://dx.doi.org/10.1016/j.atmosenv.2015.10.054 


\begin{tabular}{|c|c|c|c|c|c|c|}
\hline & & & & & $\begin{array}{l}\text { nucleation and } \\
\text { Aitken mode } \\
\text { particles and } \\
\text { in creep-idling, } \\
\text { they found } \\
\text { high PNCs in } \\
\text { nucleation and } \\
\text { around } 98 \mathrm{~nm}\end{array}$ & \\
\hline $\begin{array}{l}\text { Huang et al. } \\
\text { (2012) }\end{array}$ & $\begin{array}{l}\text { Engine } \\
\text { exhaust } \\
\text { measurem } \\
\text { ent }\end{array}$ & $\begin{array}{l}\text { Diesel bus with } \\
\text { Euro IV } \\
\text { emission } \\
\text { standards }\end{array}$ & $\begin{array}{l}5.6- \\
560\end{array}$ & EEPS 3090 & $\begin{array}{l}\text { Idling and } \\
\text { acceleration } \\
\text { was mainly } \\
\text { concentrated } \\
\text { in the } \\
\text { accumulation } \\
\text { mode particles } \\
\text { and } \\
\text { deceleration } \\
\text { was mainly } \\
\text { composed of } \\
\text { nucleation } \\
\text { mode particles }\end{array}$ & $\begin{array}{l}\text { Found } \\
\text { highest PNCs } \\
\left(5 \times 10^{8} \# \mathrm{~cm}^{-}\right. \\
\text {3) during } \\
\text { acceleration, } \\
\text { followed by } \\
\text { idling } \\
\left(2.7 \times 10^{7} \#\right. \\
\left.\mathrm{cm}^{-3}\right) \text { and } \\
\mathrm{deceleration} \\
\left(2.0 \times 10^{6} \#\right. \\
\left.\mathrm{cm}^{-3}\right)\end{array}$ \\
\hline $\begin{array}{l}\text { Karjalainen } \\
\text { et al. (2014) }\end{array}$ & $\begin{array}{l}\text { Road } \\
\text { chasing } \\
\text { and } \\
\text { Chassis } \\
\text { dynamome } \\
\text { ter }\end{array}$ & $\begin{array}{l}\text { Gasoline } \\
\text { passenger car }\end{array}$ & $\begin{array}{l}5.6- \\
520\end{array}$ & $\begin{array}{l}\text { EEPS } \\
3090, \\
\text { UCPC } \\
3025 \text { and } \\
\text { ELPI }\end{array}$ & $\begin{array}{l}\text { In both chassis } \\
\text { and on-road } \\
\text { measurements, } \\
\text { PNDs were } \\
\text { bimodal } \\
\text { during } \\
\text { acceleration } \\
\text { with modal } \\
\text { diameters of } \\
\sim 30 \text { and } 70 \\
\text { nm. During } \\
\text { accelerations, } \\
\text { the PNCs of } \\
\text { larger particles } \\
\text { were typically } \\
\text { higher than } \\
\text { those of the } \\
\text { smaller } \\
\text { particle mode. } \\
\text { In deceleration } \\
\text { conditions and } \\
\text { following idle, } \\
\text { they observed } \\
\text { unimodal } \\
\text { PNDs with } \\
\text { peak at less } \\
\text { than } 20 \text { nm in } \\
\text { diameter. }\end{array}$ & $\begin{array}{l}\text { Observed } \\
\text { highest PNCs } \\
\text { during } \\
\text { acceleration }\end{array}$ \\
\hline
\end{tabular}

Cite this article as: Goel, A., Kumar, P., 2015. Zone of influence for particle number concentrations at signalised traffic intersections, Atmospheric Environment 123, 25-38. Online Link: http://dx.doi.org/10.1016/j.atmosenv.2015.10.054 


\subsubsection{Comparison of factor contribution at different TIs}

Table 11 shows the contribution of above identified four factors to total PNCs at four different types of TIs and on the whole route. It shows that PNCs released during deceleration (26 to 78\%) contributed highest (in the $26-78 \%$ range) to total PNCs at the studied TIs, followed by acceleration (12-33\%), cruising (6-35\%) and idling (1-15\%). A strong linear correlation $\left(R^{2}=0.95\right)$ was found between average idling time and the contribution of PNCs during deceleration to the total PNC at the TIs (SI Fig. S2). Idling time was found to be directly proportional to the queue length at the TIs. Therefore, an increase in idling time is responsible for more vehicles to undergo deceleration at a TI and resulting in greater contribution from deceleration driving conditions to the total PNC.

These results are somewhat different to those reported in the literature. For instance, acceleration has been found to show highest PNCs during laboratory and on-road measurements, followed by idling and deceleration (see review of studies in Table 10). Although there are also studies (e.g. Karjalainen et al., 2014), which have reported deceleration due to engine braking to contribute significantly to ambient concentrations, but little to particle emissions, during transient driving conditions that mostly occur in the vicinity of siganlised TIs. Dispersion conditions at the TIs are hugely influenced by their geometry and the surrounding built-up environment, which contribute in diluting the emissions between the tailpipe and the sampling location (Goel and Kumar, 2014; Kumar et al., 2009). Other variables to contribute to somewhat higher contributions during deceleration could be the driving conditions and the time spent by road vehicles at the TIs. In this case, average time spent during the deceleration was $\sim 30 \%$ higher than those during acceleration. Moreover both the distance between the on-road vehicles and dilution of emissions due to lower turbulence as a aresult of decreasing vehicle speed decreases during decelebration (Carpentieri and Kumar, 2011), contributing to relatively larger share of total PNCs during deceleration.

In terms of the inter-comparability of contributions among the studied TIs, the combined contribution of deceleration, creep-idling and acceleration was highest at $\mathrm{TI}_{4 \mathrm{w}-\mathrm{nb}}(94 \%)$, followed by $\mathrm{TI}_{3 \mathrm{w}-\mathrm{wb}}(77 \%), \mathrm{TI}_{3 \mathrm{w}-\mathrm{nb}}(77 \%)$ and $\mathrm{TI}_{4 \mathrm{w} \text {-wb }}(65 \%)$. Combined factor contribution (deceleration + creep-idling + acceleration) to total PNC was 29\% Cite this article as: Goel, A., Kumar, P., 2015. Zone of influence for particle number concentrations at signalised traffic intersections, Atmospheric Environment 123, 25-38. Online Link: http://dx.doi.org/10.1016/j.atmosenv.2015.10.054 
more at $\mathrm{TI}_{4 \mathrm{w}-\mathrm{nb}}$ as compare to $\mathrm{TI}_{4 \mathrm{w} \text {-wb }}$ while no such differences were observed between $\mathrm{TI}_{3 \mathrm{w}-\mathrm{nb}}$ and $\mathrm{TI}_{3 \mathrm{w} \text {-wb }}$ (Table 11).

Table 11. Factor contribution in percentage to total PNC on whole route and at four different types of TIs.

\begin{tabular}{lllll}
\hline & $\begin{array}{l}\text { Factor 1 } \\
\text { (Deceleration) }\end{array}$ & $\begin{array}{l}\text { Factor 2 } \\
\text { (Acceleration) }\end{array}$ & $\begin{array}{l}\text { Factor 3 } \\
\text { (Cruising) }\end{array}$ & $\begin{array}{l}\text { Factor 4 } \\
\text { (Creep-idling) }\end{array}$ \\
\hline $\mathrm{TI}_{4 \mathrm{w}-\mathrm{nb}}$ & 78 & 12 & 6 & 4 \\
\hline $\mathrm{TI}_{4 \mathrm{w}-\mathrm{wb}}$ & 26 & 24 & 35 & 15 \\
\hline $\mathrm{TI}_{3 \mathrm{w}-\mathrm{nb}}$ & 49 & 21 & 23 & 7 \\
\hline $\mathrm{TI}_{3 \mathrm{w}-\mathrm{wb}}$ & 38 & 33 & 23 & 6 \\
\hline Average at all & 48 & 23 & 22 & 8 \\
types of TIs & & & & 10 \\
\hline Whole Route & 33 & 28 & 29 & \\
\hline
\end{tabular}

\section{Summary, conclusions and future work}

This study presents the longitudinal distances, representing ZoI around a TI, at four different types of TIs under three different driving conditions. ZoI of a TI was estimated on the basis of on-road PNCs and driving speed with respect to distance from the centre of a TI at different TIs. These on-road PNCs were obtained by mobile monitoring of particle number and size distribution in 5-560 $\mathrm{nm}$ size ranges. The results are discussed in terms of ZoI of a TI for PNC. Generalised profiles of the PNCs with respect to distance within the ZoI is also presented. Furthermore, contribution of PNC due to different driving conditions such as deceleration, acceleration, creep-idling and cruising to overall PNC within ZoI of TIs is also estimated by using the PMF modelling.

The following conclusions were drawn:

- Irrespective of a type of a TI and driving conditions, length of ZoI at a TI was found to depend on acceleration, deceleration and idling time of a vehicle.

Cite this article as: Goel, A., Kumar, P., 2015. Zone of influence for particle number concentrations at signalised traffic intersections, Atmospheric Environment 123, 25-38. Online Link: http://dx.doi.org/10.1016/j.atmosenv.2015.10.054 
Maximum length of ZoI correlated linearly with the idling time at a TI while average length of ZoI is found to have a linear correlation with acceleration.

- During stop and go driving conditions, maximum length of ZoI was found to be the largest $(379 \mathrm{~m})$ at $\mathrm{TI}_{4 \mathrm{w}-\mathrm{nb}}$, followed by $\mathrm{TI}_{3 \mathrm{w}-\mathrm{wb}}(297 \mathrm{~m}), \mathrm{TI}_{3 \mathrm{w}-\mathrm{nb}}(268 \mathrm{~m})$ and $\mathrm{TI}_{4 \mathrm{w}-\mathrm{wb}}(120 \mathrm{~m})$. This indicates that receptors loacted even at a farther distance from the TI will be exposed to peak PNCs emitted due to presence of a traffic signal at $\mathrm{TI}_{4 \mathrm{w}-\mathrm{nb}}$.

- During multiple stopping driving conditions, ZoI of a $\mathrm{TI}_{4 \mathrm{w}-\mathrm{nb}}$ was almost similar to those found in stop and go driving conditions. This indicates that maixmum length of $\mathrm{ZoI}$ at $\mathrm{TI}_{4 \mathrm{w}-\mathrm{nb}}$ is not affected by driving conditions. As expected, the ZoI shrinked to almost zero in case of a free-flow driving conditions as there was not much variations in driving speed $\left(30 \pm 6 \mathrm{~km} \mathrm{~h}^{-1}\right)$ of the vehicle, which resulted in almost constant PNCs with respect to changing distnace from the centre of a TI.

- A three degree polynomial fit represented the PNC well within the ZoI. At all four types of TIs, traffic volume influenced the coefficient of polynomial fit. Delay time played an important role in determining the coefficients at four-way TIs while wind speed at three-way TIs.

- The results of PMF modelling suggested that, irrespective of any type of a TI, average contribution towards PNCs was highest during deceleration (48 $\pm 22 \%$ ), followed by acceleration $(23 \pm 9 \%)$, cruising $(22 \pm 12 \%)$ and idling $(8 \pm 5 \%)$. It indicates that change in driving conditions of road vehicles due to the presence of traffic signals is a major cause for higher PNCs at TIs.

We presented ZoI and contribution of different driving conditions at TIs for the first time. This work also opened up a number of questions for further research. For example, estimating the height of ZoI is important to represent the exposure of receptors residing at different height near the TI and could be considered as a part of future studies. Further, length of ZoI is estimated for selected traffic periods at TIs and extending the study for off-peak traffic periods could be interesting to understand the changes in the length of ZoI. This study also interpreted profiles of PNC within ZoI in terms of physically known parameters based on dimensional analysis. Further field

Cite this article as: Goel, A., Kumar, P., 2015. Zone of influence for particle number concentrations at signalised traffic intersections, Atmospheric Environment 123, 25-38. Online Link: http://dx.doi.org/10.1016/j.atmosenv.2015.10.054 
measurements are recommended to generalise our results to TIs in diverse geographical settings elsewhere.

\section{Acknowledgements}

Authors thank the UK Commonwealth Commission, Prof Alan Robins, Mr Shobhan Navaratnarajah, Mr Mihai Pop, Mr Ganesh Chandrashekran, Mr Santosh Tirunagari and the Department of Civil \& Environmental Engineering at the University of Surrey, UK, for their help during experiments and discussion. PK thanks the funding received from the University Global Partnership Network (UGPN) through the project "Comparison of Air Pollution in Transportation ENvironments (CAPTEN): Development and Demonstration Based on Selected UK and US Cities" for supporting this research work.

\section{References}

Al-Dabbous, A.N., Kumar, P., 2014. The influence of roadside vegetation barriers on airborne nanoparticles and pedestrians exposure under varying wind conditions. Atmospheric Environment 90, 113-124.

Al-Dabbous, A.N., Kumar, P., 2015. Source apportionment of airborne nanoparticles in a Middle Eastern city using positive matrix factorization. Environmental Science: Processes \& Impacts 17, 802-812.

Batterman, S.A., Zhang, K., Kononowech, R., 2010. Prediction and analysis of near-road concentrations using a reduced-form emission/dispersion model. Environmental Health 9, 29-29.

Bos, I., Jacobs, L., Nawrot, T.S., de Geus, B., Torfs, R., Int Panis, L., Degraeuwe, B., Meeusen, R., 2011. No exercise-induced increase in serum BDNF after cycling near a major traffic road. Neuroscience Letters 500, 129-132.

Carpentieri, M., Kumar, P., 2011. Ground-fixed and on-board measurements of nanoparticles in the wake of a moving vehicle. Atmospheric Environment 45, 5837-5852.

Dirks, K.N., Johns, M.D., Hay, J.E., Sturman, A.P., 2003. A semi-empirical model for predicting the effect of changes in traffic flow patterns on carbon monoxide concentrations. Atmospheric Environment 37, 2719-2724.

Cite this article as: Goel, A., Kumar, P., 2015. Zone of influence for particle number concentrations at signalised traffic intersections, Atmospheric Environment 123, 25-38. Online Link: http://dx.doi.org/10.1016/j.atmosenv.2015.10.054 
Domínguez-Sáez, A., Viana, M., Barrios, C.C., Rubio, J.R., Amato, F., Pujadas, M., Querol, X., 2012. Size-resolved particle number emission patterns under real-world driving conditions using positive matrix factorization. Environmental Science \& Technology 46, 11187-11194.

DoT, 2013. Department of transport. Annual average daily traffic flow at 351 point in Surrey. http://www.dft.gov.uk/traffic-counts/cp.php?la=Surrey accessed on $10 / 07 / 2014$.

Fruin, S., Westerdahl, D., Sax, T., Sioutas, C., Fine, P.M., 2008. Measurements and predictors of on-road ultrafine particle concentrations and associated pollutants in Los Angeles. Atmospheric Environment 42, 207-219.

Fujitani, Y., Kumar, P., Tamura, K., Fushimi, A., Hasegawa, S., Takahashi, K., Tanabe, K., Kobayashi, S., Hirano, S., 2012. Seasonal differences of the atmospheric particle size distribution in a metropolitan area in Japan. Science of the Total Environment 437, 339-347.

Goel, A., Kumar, P., 2014. A review of fundamental drivers governing the emissions, dispersion and exposure to vehicle-emitted nanoparticles at signalised traffic intersections. Atmospheric Environment 97, 316-331.

Goel, A., Kumar, P., 2015. Characterisation of nanoparticle emissions and exposure at traffic intersections through fast-response mobile and sequential measurements. Atmospheric Environment 107, 374-390.

Gokhale, S., Khare, M., 2005. A hybrid model for predicting carbon monoxide from vehicular exhausts in urban environments. Atmospheric Environment 39, 40254040.

Gokhale, S., Raokhande, N., 2008. Performance evaluation of air quality models for predicting PM10 and PM2.5 concentrations at urban traffic intersection during winter period. Science of the total environment 394, 9-24.

Gupta, I., Salunkhe, A., Kumar, R., 2012. Source apportionment of PM(10) by positive matrix factorization in urban area of Mumbai, India. The Scientific World Journal $2012,585791$.

Harrison, R.M., Beddows, D.C.S., Dall'Osto, M., 2011. PMF analysis of wide-range particle size spectra collected on a major highway. Environment Science \& Technology 45, 5522-5528.

Cite this article as: Goel, A., Kumar, P., 2015. Zone of influence for particle number concentrations at signalised traffic intersections, Atmospheric Environment 123, 25-38. Online Link: http://dx.doi.org/10.1016/j.atmosenv.2015.10.054 
He, H.-d., Lu, W.-Z., Xue, Y., 2009. Prediction of PM10 concentrations at urban traffic intersections using semi-empirical box modelling with instantaneous velocity and acceleration. Atmospheric Environment 43, 6336-6342.

Holder, A.L., Hagler, G.S.W., Yelverton, T.L.B., Hays, M.D., 2014. On-road black carbon instrument intercomparison and aerosol characteristics by driving environment. Atmospheric Environment 88, 183-191.

Holmes, N.S., Morawska, L., Mengersen, K., Jayaratne, E., 2005. Spatial distribution of submicrometre particles and $\mathrm{CO}$ in an urban microscale environment. Atmospheric Environment 39, 3977-3988.

Hopke, P.K., 2000. A guide to positive matrix factorization, Workshop on UNMIX and PMF as Applied to PM2, p. 600.

Huang, C., Lou, D., Hu, Z., Tan, P., Yao, D., Hu, W., Li, P., Ren, J., Chen, C., 2012. Ultrafine particle emission characteristics of diesel engine by on-board and test bench measurement. Journal of Environmental Sciences 24, 1972-1978.

Hudda, N., Gould, T., Hartin, K., Larson, T.V., Fruin, S.A., 2014. Emissions from an international airport increase particle number concentrations 4-fold at $10 \mathrm{~km}$ downwind. Environmental Science \& Technology 48, 6628-6635.

Hudda, N., Kostenidou, E., Sioutas, C., Delfino, R.J., Fruin, S.A., 2011. Vehicle and driving characteristics that influence in-cabin particle number concentrations. Environmental Science \& Technology 45, 8691-8697.

Imhof, D., Weingartner, E., Ordóñez, C., Gehrig, R., Hill, M., Buchmann, B., Baltensperger, U., 2005. Real-world emission factors of fine and ultrafine aerosol particles for different traffic situations in Switzerland. Environmental Science \& Technology 39, 8341-8350.

Jacobs, L., Nawrot, T., de Geus, B., Meeusen, R., Degraeuwe, B., Bernard, A., Sughis, M., Nemery, B., Panis, L., 2010. Subclinical responses in healthy cyclists briefly exposed to traffic-related air pollution: an intervention study. Environmental Health 9, 64.

Joodatnia, P., Kumar, P., Robins, A., 2013a. The behaviour of traffic produced nanoparticles in a car cabin and resulting exposure rates. Atmospheric Environment 65, 40-51.

Cite this article as: Goel, A., Kumar, P., 2015. Zone of influence for particle number concentrations at signalised traffic intersections, Atmospheric Environment 123, 25-38. Online Link: http://dx.doi.org/10.1016/j.atmosenv.2015.10.054 
Joodatnia, P., Kumar, P., Robins, A., 2013b. Fast response sequential measurements and modelling of nanoparticles inside and outside a car cabin. Atmospheric Environment 71, 364-375.

JRC 2009. Positive matrix factorisation: an introduction to the chemometric evaluation of environmental monitoring data using PMF. http://publications.jrc.ec.europa.eu/repository/bitstream/JRC52754/reqno_jrc52 754_final_pdf_version\%5B1\%5D.pdf (accessed on 7th May 2015).

Karjalainen, P., Pirjola, L., Heikkilä, J., Lähde, T., Tzamkiozis, T., Ntziachristos, L., Keskinen, J., Rönkkö, T., 2014. Exhaust particles of modern gasoline vehicles: A laboratory and an on-road study. Atmospheric Environment 97, 262-270.

Kastner-Klein, P., Fedorovich, E., Ketzel, M., Berkowicz, R., Britter, R., 2003. The modelling of turbulence from traffic in urban dispersion models - Part II: evaluation against laboratory and full-scale concentration measurements in street canyons. Environmental Fluid Mechanics 3, 145-172.

Kim, K.H., Lee, S.-B., Woo, S.H., Bae, G.-N., 2014. NOx profile around a signalized intersection of busy roadway. Atmospheric Environment 97, 144-154.

Knibbs, L.D., de Dear, R.J., Morawska, L., 2010. Effect of cabin ventilation rate on ultrafine particle exposure inside automobiles. Environmental Science \& Technology 44, 3546-3551.

Kumar, P., Fennell, P., Britter, R., 2008. Measurements of particles in the 5-1000 nm range close to road level in an urban street canyon. Science of the Total Environment 390, 437-447.

Kumar, P., Robins, A., Britter, R., 2009. Fast response measurements of the dispersion of nanoparticles in a vehicle wake and a street canyon. Atmospheric Environment 43, 6110-6118.

Kumar, P., Ketzel, M., Vardoulakis, S., Pirjola, L., Britter, R., 2011. Dynamics and dispersion modelling of nanoparticles from road traffic in the urban atmospheric environment-A review. Journal of Aerosol Science 42, 580-603.

Kumar, P., Robins, A., Vardoulakis, S., Britter, R., 2010. A review of the characteristics of nanoparticles in the urban atmosphere and the prospects for developing regulatory controls. Atmospheric Environment 44, 5035-5052.

Cite this article as: Goel, A., Kumar, P., 2015. Zone of influence for particle number concentrations at signalised traffic intersections, Atmospheric Environment 123, 25-38. Online Link: http://dx.doi.org/10.1016/j.atmosenv.2015.10.054 
Kumar , P., Pirjola, L., Ketzel, M., Harrison, R.M., 2013. Nanoparticle emissions from 11 non-vehicle exhaust sources- A review. Atmospheric Environment 67, 252-277.

Kwak, J.H., Kim, H.S., Lee, J.H., Lee, S.H., 2014. On-road chasing measurement of exhaust particle emissions from diesel, CNG, LPG, and DME-fueled vehicles using a mobile emission laboratory. International Journal of Automotive Technology 15, 543-551.

Metoffice, 2014. UK met office. http://www.metoffice.gov.uk/datapoint (accessed on 8 March 2014).

Mishra, V.K., Padmanabhamutry, B., 2003. Performance evaluation of CALINE3, CAL3QHC and PART5 in predicting lead concentration in the atmosphere over Delhi. Atmospheric Environment 37, 3077-3089.

Morawska, L., Thomas, S., Hofmann, W., Ristovski, Z., Jamriska, M., Rettenmoser, T., Kagerer, S., 2004. Exploratory cross-sectional investigations on ambient submicrometer particles in Salzburg, Austria. Atmospheric Environment 38, 35293533.

Oladnia, S., Saeedi, M., Bazkiaei, A.R., 2008. Intersection air pollution emission prediction using software HYROAD: A casestudy Fatemi-Valiasr intersection from Iran, World Environmental and Water Resources Congress 2008. American Society of Civil Engineers, pp. 1-11.

Patton, A.P., Collins, C., Naumova, E.N., Zamore, W., Brugge, D., Durant, J.L., 2014. An hourly regression model for ultrafine particles in a near-highway urban area. Environmental Science \& Technology 48, 3272-3280.

Ritner, M., Westerlund, K.K., Cooper, C.D., Claggett, M., 2013. Accounting for acceleration and deceleration emissions in intersection dispersion modeling using MOVES and CAL3QHC. Journal of the Air \& Waste Management Association 63, 724-736.

Shah, S.D., Cocker, D.R., 2005. A fast scanning mobility particle spectrometer for monitoring transient particle size distributions. Aerosol Science and Technology 39, 519-526.

Tsang, H., Kwok, R., Miguel, A.H., 2008. Pedestrian exposure to ultrafine particles in Hong Kong under heavy traffic conditions. Aerosol and Air Quality Research 8, 1927.

Cite this article as: Goel, A., Kumar, P., 2015. Zone of influence for particle number concentrations at signalised traffic intersections, Atmospheric Environment 123, 25-38. Online Link: http://dx.doi.org/10.1016/j.atmosenv.2015.10.054 
Vogt, R., Scheer, V., Casati, R., Benter, T., 2003. On-road measurement of particle emission in the exhaust plume of a diesel passenger car. Environmental Science \& Technology 37, 4070-4076.

Wang, F., Ketzel, M., Ellermann, T., Wåhlin, P., Jensen, S.S., Fang, D., Massling, A., 2010. Particle number, particle mass and NOx emission factors at a highway and an urban street in Copenhagen. Atmospheric Chemistry and Physics 10, 2745-2764.

Wang, J., Storey, J., Domingo, N., Huff, S., Thomas, J., West, B., 2006. Studies of diesel engine particle emissions during transient operations using an engine exhaust particle sizer. Aerosol Science and Technology 40, 1002-1015.

Wang, Y., Zhu, Y., Salinas, R., Ramirez, D., Karnae, S., John, K., 2008. Roadside measurements of ultrafine particles at a busy urban intersection. Journal of the Air \& Waste Management Association 58, 1449-1457.

Zhu, Y., Eiguren-Fernandez, A., Hinds, W.C., Miguel, A.H., 2007. In-cabin commuter exposure to ultrafine particles on Los Angeles freeways. Environmental Science \& Technology 41, 2138-2145.

Cite this article as: Goel, A., Kumar, P., 2015. Zone of influence for particle number concentrations at signalised traffic intersections, Atmospheric Environment 123, 25-38. Online Link: http://dx.doi.org/10.1016/j.atmosenv.2015.10.054 


\title{
Supplementary Material
}

\section{Zone of influence for particle number concentrations at signalised traffic intersections}

\author{
Anju Goela, Prashant Kumara, b, 2 \\ aDepartment of Civil and Environmental Engineering, Faculty of Engineering and Physical \\ Sciences, University of Surrey, Guildford GU2 7XH, United Kingdom \\ bEnvironmental Flow (EnFlo) Research Centre, Faculty of Engineering and Physical \\ Sciences, University of Surrey, Guildford GU2 7XH, United Kingdom
}

\section{S1. Dimensional analysis of PNCs profiles in ZoI}

An attempt was made to represent the coefficient of the polynomial Eq. (S1) in physically known parameters such as particle number emission factor (PNF) (\# $\mathrm{cm}^{-2} \mathrm{~s}^{-}$ $\left.{ }^{1}\right)$, wind speed $(\mathrm{u})\left(\mathrm{m} \mathrm{s}^{-1}\right)$, average driving speed $(\mathrm{v})\left(\mathrm{m} \mathrm{s}^{-1}\right)$, average acceleration or deceleration with in ZoI $\left(\mathrm{m} \mathrm{s}^{-2}\right)$, number of lanes at a TI, delay time at a TI (t) (s), width of the roads intersecting at a TI (m) and height of buildings around a TI (h) at a TI based on dimensional analysis.

Generalised form of polynomial equation is:

$$
(P N C)_{x}=a x^{3}+b x^{2}+c x+d
$$

Based on the dimensional equality, the dimension of $a, b, c$ and $d$ should be $\# \mathrm{~cm}^{-6}, \# \mathrm{~cm}^{-}$ 5 , \# $\mathrm{cm}^{-4}$, and $\# \mathrm{~cm}^{-3}$. By, replacing coefficients with their dimensions, Eq. (S1) becomes:

\footnotetext{
${ }^{2}$ Corresponding author: Department of Civil and Environmental Engineering, Faculty of Engineering and Physical Sciences, University of Surrey, Guildford GU2 7XH, United Kingdom; Tel.: +44 1483 682762; Fax: +44 1483 682135; E-mail addresses: P.Kumar@surrey.ac.uk,Prashant.Kumar@cantab.net

Cite this article as: Goel, A., Kumar, P., 2015. Zone of influence for particle number concentrations at signalised traffic intersections, Atmospheric Environment 123, 25-38. Online Link: http://dx.doi.org/10.1016/j.atmosenv.2015.10.054
} 
$\left(\# \mathrm{~cm}^{-3}\right)=\left(\# \mathrm{~cm}^{-6}\right) \mathrm{x}^{3}+\left(\# \mathrm{~cm}^{-5}\right) \mathrm{x}^{2}+\left(\# \mathrm{~cm}^{-4}\right) \mathrm{x}+\left(\# \mathrm{~cm}^{-3}\right)$

Therefore, $d$ has same dimensions as of PNC and was found to be equal to the $70^{\text {th }}$ percentile of measured PNC with in ZoI. Taking the coefficients of same dimension on one side the Eq. (S2) becomes:

$\left(\# \mathrm{~cm}^{-3}\right)-\left(\# \mathrm{~cm}^{-3}\right)=\left(\# \mathrm{~cm}^{-6}\right) \mathrm{x}^{3}+\left(\# \mathrm{~cm}^{-5}\right) \mathrm{x}^{2}+\left(\# \mathrm{~cm}^{-4}\right) \mathrm{x}$

The maximum dimension that is common among $a, b$ and $c$ is $\# \mathrm{~cm}^{-4}$. Therefore Eq. (S3) can be re-written as Eq. (S4):

$(\mathrm{PNC})_{\mathrm{x}}-\left(\# \mathrm{~cm}^{-3}\right)=\left(\# \mathrm{~cm}^{-4}\right)\left[\left(\mathrm{cm}^{-2}\right) \mathrm{x}^{3}+\left(\mathrm{cm}^{-1}\right) \mathrm{x}^{2}+\mathrm{x}\right]$

The possible combination of different factors for $\# \mathrm{~cm}^{-4}$ is presented in Eq. (S5):

$(c)\left(\# c m^{-4}\right)=(P N F)\left(\# s^{-1} c^{-2}\right) \times\left(\frac{100}{w}\right)\left(c^{-1} s\right) \times\left(\frac{10^{4}}{h}\right)\left(c^{-1}\right)$

Where $\mathrm{w}$ is the resultant wind speed in $\mathrm{m} \mathrm{s}^{-1}$ and is given by Eq. (S6)

$\mathrm{w}=\sqrt[2]{\left(\alpha \times \mathrm{u}^{2}+\beta \times \mathrm{v}^{2}\right)}$

Where ' $\alpha$ ' and ' $\beta$ ' are constants that depends on traffic volume and built-up area around a TI.

Except ' $w$ ', all other factors in the Eq. (S5) can be considered constant for different runs at any TI. If the above proposed combination of factors for coefficient ' $c$ ' is valid, a correlation should exist for a unique value of $\alpha$ and $\beta$ between $c$ and $w^{-1}$ corresponding to different runs at any TI. This assumption is checked for different runs at a $\mathrm{TI}_{4 \mathrm{w}-\mathrm{nb} \text {. To }}$ start with, values of $\alpha$ and $\beta$ were considered same as proposed by Kastner-Klein et al. (2003) for a street canyon. The $\alpha$ was considered as $1 \times 10^{-2}$ and $\beta$ was considered as $1.44 \times 10^{-5} \times(\mathrm{k})^{(2 / 3)}$, where $k$ is the traffic density (veh $\mathrm{km}^{-1}$ ). Vehicle per kilometre length of a TI was estimated by considering all vehicles as a car of average length $3 \mathrm{~m}$ and a safe distance of $3 \mathrm{~m}$ between two adjacent vehicles at a four lane road. Total length of the road was considered as $80 \mathrm{~m}$ i.e., the length of $\mathrm{ZoI}$ of TI $4 \mathrm{w}$-nb on either side of a TI. Estimated vehicular density at a $\mathrm{TI}_{4 \mathrm{w}-\mathrm{nb}}$, was 44 vehicles per metre. Then values of $\alpha$ and $\beta$ were then varied to get a good correlation between $c$ and $w^{-1}$. In total, we tried 220 combinations of $\alpha$ and $\beta$, and a $\mathrm{R}^{2}=0.8$ was found between $c$ and $w^{-1}$ for a Cite this article as: Goel, A., Kumar, P., 2015. Zone of influence for particle number concentrations at signalised traffic intersections, Atmospheric Environment 123, 25-38. Online Link: http://dx.doi.org/10.1016/j.atmosenv.2015.10.054 
unique $\alpha=0.002$ and $\beta=5.15 \times 10^{-4}$ at a $\mathrm{TI}_{4 \mathrm{w}-\mathrm{nb}}$. It proves the validity of the proposed combination of factors for the coefficient $c$ in the Eq. (S1).

The possible combination of various factors at a TI for a modified coefficient $\left(\mathrm{cm}^{-2}\right)$ of $x^{3}$ in the Eq. (S4) could be presented by Eq. (S7):

$\frac{\mathrm{a}}{\mathrm{c}}\left(\mathrm{cm}^{-2}\right)=\left(\frac{10^{2}}{u}\right)\left(\mathrm{s} \mathrm{cm}^{-1}\right) \times\left(\frac{10^{2}}{v}\right)\left(\mathrm{s} \mathrm{cm}^{-1}\right) \times t^{-2}\left(\mathrm{~s}^{-2}\right)$

To check the validity of the proposed combination, a regression was performed between $a$ divided by $c$ and the result of Eq.S7 for different runs at a TI $\mathrm{TI}_{4 \mathrm{w}-\mathrm{nb}}$. A good correlation with $\mathrm{R}^{2}=0.87$ was found that proves the validity of the proposed combination of factors. In addition to this combination, we also tried replacing, wind speed with driving speed and resultant wind speed but a poor $R^{2}=0.10$ was found in both the cases.

The possible combination of factors at a TI for third and last modified coefficient $\left(\mathrm{cm}^{-1}\right)$ for $x$ in the Eq. (S4) is shown in Eq. (S8):

$\frac{b}{c}\left(\mathrm{~cm}^{-1}\right)=\left(\frac{10^{2}}{u}\right)\left(\mathrm{cm}^{-1} \mathrm{~s}\right) \times t^{-1}\left(\mathrm{~s}^{-1}\right)$

Again, to check the validity of the Eq. (S8), results of Eq. (S8) were regressed with coefficient $b$ divided by coefficient $c$ for various runs at a $\mathrm{TI}_{4 \mathrm{w}-\mathrm{nb}}$. A $\mathrm{R}^{2}=0.49$ was found that proves the validity of Eq. (S8). In addition to combination proposed in Eq. (S8), other two combinations (i) by replacing wind speed with resultant speed and (ii) by replacing wind speed with driving speed were also tried and $\mathrm{R}^{2}$ values of 0.28 and 0.03 were found for combination (i) and (ii) respectively. Based on Eq. (S5), (S7) and (S8), Eq. (S1) can be rewritten as Eq. (S9):

$(\mathrm{PNC})_{\mathrm{x}}-\mathrm{d}=(\mathrm{PNF}) \times\left(\frac{1}{\mathrm{w}}\right) \times\left(\frac{1}{\mathrm{~h}}\right)\left\{\left(\frac{1}{\mathrm{u}}\right) \times\left(\frac{1}{v}\right) \times \mathrm{t}^{-2} \mathrm{x}^{3}+\left(\frac{1}{\mathrm{u}}\right) \times \mathrm{t}^{-1} \mathrm{x}^{2}+\mathrm{x}\right\}$

Cite this article as: Goel, A., Kumar, P., 2015. Zone of influence for particle number concentrations at signalised traffic intersections, Atmospheric Environment 123, 25-38. Online Link: http://dx.doi.org/10.1016/j.atmosenv.2015.10.054 

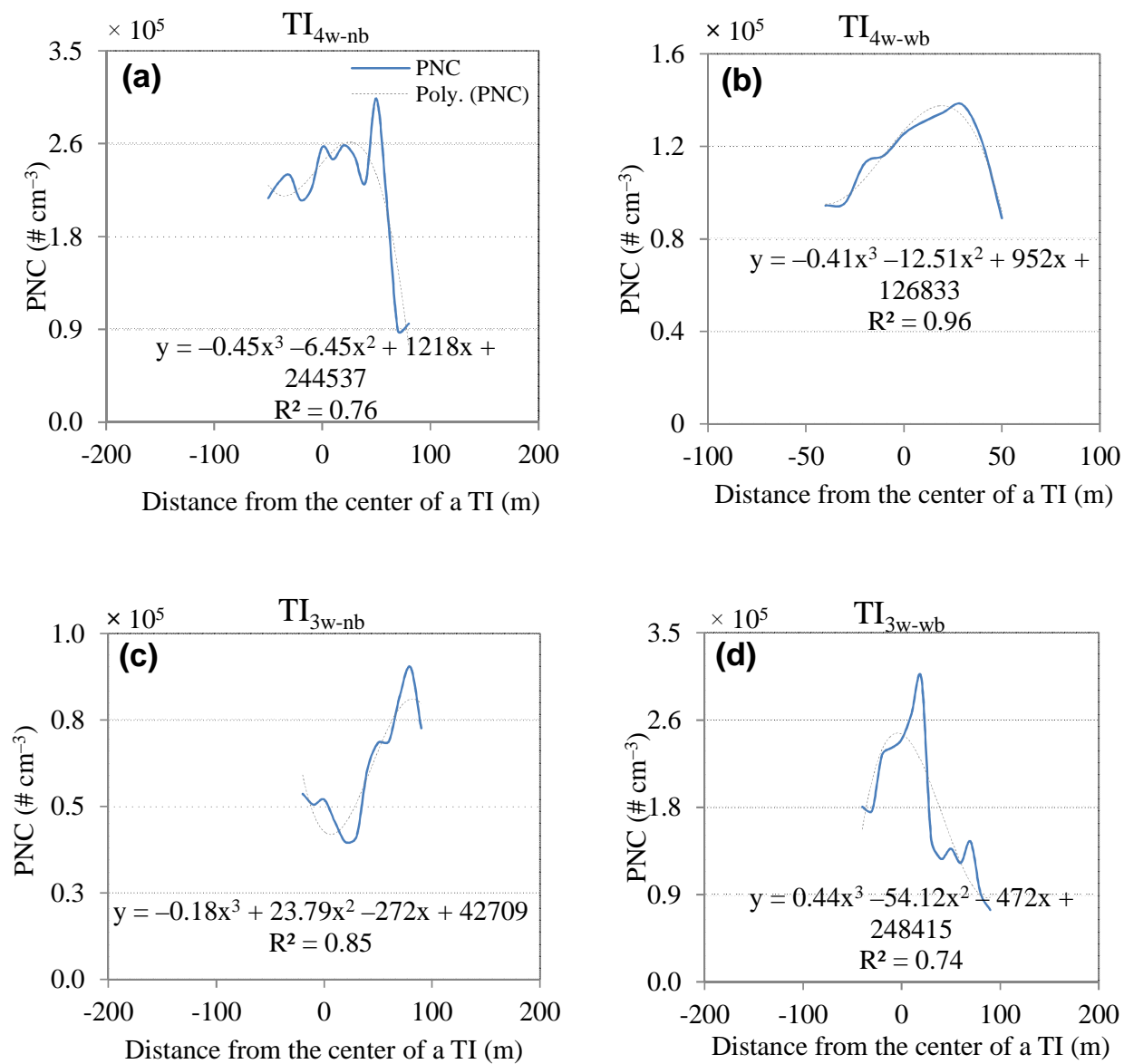

Fig. S1. PNC profiles with respect to distance from the centre of a TI within ZoI at (a) $\mathrm{TI}_{4 \mathrm{w}-\mathrm{nb},(\mathrm{b})} \mathrm{TI}_{4 \mathrm{w}-\mathrm{wb}},(\mathrm{c}) \mathrm{TI}_{3 \mathrm{w}-\mathrm{nb}}$ and (d) $\mathrm{TI}_{3 \mathrm{w}-\mathrm{wb}}$.

Cite this article as: Goel, A., Kumar, P., 2015. Zone of influence for particle number concentrations at signalised traffic intersections, Atmospheric Environment 123, 25-38. Online Link: http://dx.doi.org/10.1016/j.atmosenv.2015.10.054 


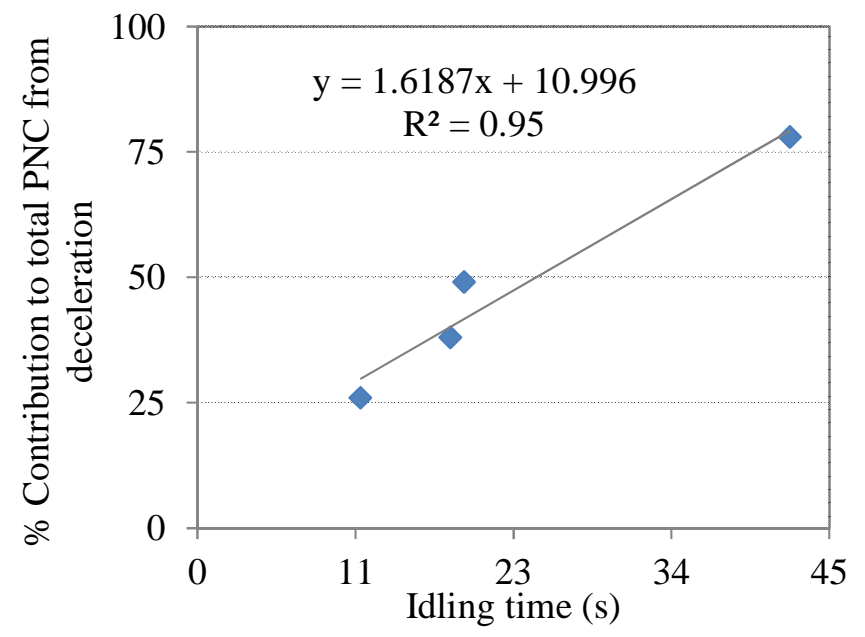

Fig. S2. Correlation between factor contribution of deceleration and average idling time a TI.

Cite this article as: Goel, A., Kumar, P., 2015. Zone of influence for particle number concentrations at signalised traffic intersections, Atmospheric Environment 123, 25-38. Online Link: http://dx.doi.org/10.1016/j.atmosenv.2015.10.054 
Table S1. Starting and end points i.e., $X_{1}$ and $X_{2}$ of ZoI of four different types of TIs in stop and go driving condition. In this table values of $X_{1}$ and $X_{2}$ are shown only for the runs where PNC versus distance profile and driving speed versus distance profile of a TI intersected at two points.

\begin{tabular}{|c|c|c|c|c|c|c|c|c|c|c|c|c|c|c|c|c|c|c|c|c|}
\hline \multirow[t]{2}{*}{ Run No } & \multicolumn{2}{|c|}{$\mathrm{TI}_{1}$} & \multicolumn{2}{|c|}{$\mathrm{TI}_{2}$} & \multicolumn{2}{|c|}{$\mathrm{TI}_{3}$} & \multicolumn{2}{|c|}{$\mathrm{TI}_{4}$} & \multicolumn{2}{|c|}{$\mathrm{TI}_{5}$} & \multicolumn{2}{|c|}{$\mathrm{TI}_{6}$} & \multicolumn{2}{|c|}{$\mathrm{TI}_{7}$} & \multicolumn{2}{|c|}{$\mathrm{TI}_{8}$} & \multicolumn{2}{|c|}{$\mathrm{TI}_{9}$} & \multicolumn{2}{|c|}{$\mathrm{TI}_{10}$} \\
\hline & $X_{1}$ & $X_{2}$ & $X_{1}$ & $X_{2}$ & $X_{1}$ & $X_{2}$ & $X_{1}$ & $X_{2}$ & $X_{1}$ & $X_{2}$ & $X_{1}$ & $X_{2}$ & $X_{1}$ & $X_{2}$ & $X_{1}$ & $X_{2}$ & $X_{1}$ & $X_{2}$ & $X_{1}$ & $X_{2}$ \\
\hline R1 & 29 & -11 & 90 & -120 & - & - & 19 & -120 & - & - & - & - & 19 & -94 & - & - & - & - & - & - \\
\hline R2 & 117 & -27 & - & - & - & - & 189 & -104 & - & - & 104 & 37 & - & - & - & - & - & - & 80 & -40 \\
\hline R3 & 84 & -102 & - & - & 70 & -51 & - & - & 136 & -113 & - & - & 52 & 17 & 80 & -60 & - & - & - & - \\
\hline $\mathrm{R} 4$ & - & - & 97 & -20 & 50 & -50 & 109 & 14 & 95 & -115 & - & - & - & - & - & - & 45 & -20 & - & - \\
\hline R5 & - & - & 95 & 54 & 7 & 13 & 142 & -6 & 65 & -83 & - & - & - & - & - & - & - & - & 40 & -40 \\
\hline R6 & - & - & - & - & - & - & - & - & - & - & - & - & - & - & - & - & 75 & 12 & - & - \\
\hline R7 & 102 & -30 & - & - & - & - & - & - & - & - & - & - & - & - & - & - & 137 & 98 & - & - \\
\hline R8 & 63 & -91 & - & - & - & - & 65 & 37 & - & - & - & - & 107 & 49 & - & - & - & - & - & - \\
\hline R9 & - & - & - & - & - & - & 83 & -18 & - & - & - & - & 24 & -35 & - & - & 22 & -91 & 50 & -30 \\
\hline R10 & - & - & 79 & -39 & - & - & - & - & - & - & - & - & - & - & - & - & - & - & 50 & -40 \\
\hline R11 & 16 & -48 & 27 & -33 & - & - & 46 & -15 & - & - & - & - & - & - & - & - & 42 & -45 & - & - \\
\hline R12 & 66 & -7 & 30 & -80 & - & - & 75 & 27 & - & - & - & - & 89 & -38 & - & - & - & - & 10 & -20 \\
\hline R13 & - & - & - & - & - & - & 116 & -33 & 86 & -151 & - & - & - & - & - & - & - & - & - & - \\
\hline R14 & - & - & - & - & - & - & - & - & - & - & - & - & - & - & 40 & -30 & 84 & -48 & - & - \\
\hline R15 & - & - & 30 & -60 & - & - & 34 & -78 & - & - & 40 & -154 & 25 & -111 & - & - & 60 & -55 & - & - \\
\hline R16 & - & - & - & - & - & - & 0 & -190 & 109 & -56 & - & - & 157 & 58 & - & - & 142 & -34 & - & - \\
\hline R17 & - & - & 140 & 0 & - & - & 141 & -73 & 102 & -141 & 117 & -137 & - & - & - & - & - & - & - & - \\
\hline R18 & 104 & -18 & - & - & 80 & 50 & 140 & 70 & - & - & 78 & 29 & - & - & 90 & 30 & - & - & - & - \\
\hline R19 & - & - & 30 & -50 & 90 & 20 & - & - & - & - & 123 & 21 & - & - & - & - & - & - & 70 & -20 \\
\hline R20 & 33 & -43 & 70 & -20 & - & - & - & - & 129 & -46 & - & - & 116 & 30 & - & - & - & - & 20 & -40 \\
\hline R21 & 62 & -143 & 50 & -70 & - & - & 153 & 99 & - & - & - & - & 121 & -32 & - & - & - & - & - & - \\
\hline R22 & - & - & 60 & -120 & - & - & 59 & -17 & 146 & -77 & - & - & - & - & - & - & - & - & - & - \\
\hline Max & 117 & -143 & 140 & -120 & 90 & -51 & 189 & -190 & 146 & -151 & 123 & -154 & 157 & -111 & 90 & -60 & 142 & -98 & 80 & -40 \\
\hline Min & 16 & -7 & 27 & $\mathbf{0}$ & 50 & 20 & $\mathbf{0}$ & -6 & 65 & 14 & 40 & 21 & 19 & 17 & 40 & 30 & 22 & 12 & 10 & -20 \\
\hline Average & 68 & -52 & 67 & -47 & 59 & -4 & 91 & -27 & 109 & -98 & 92 & -41 & 79 & -17 & 70 & -20 & 76 & -23 & 46 & -33 \\
\hline Stdev & 34 & 45 & 35 & 49 & 33 & 45 & 55 & 76 & 27 & 38 & 34 & 96 & 50 & 60 & 26 & 46 & 44 & 57 & 25 & 10 \\
\hline Median & 63 & -42 & 65 & -45 & 70 & 13 & 83 & -17 & 106 & -98 & 104 & 21 & 89 & -32 & 80 & -30 & 68 & -40 & 50 & -40 \\
\hline
\end{tabular}


Table S2. Starting and end points i.e., $X_{1}$ and $X_{2}$ of ZoI of four different types of TIs in multiple stopping driving condition. In this table values of $X_{1}$ and $X_{2}$ are shown only for the runs where PNC versus distance profile and driving speed versus distance profile of a TI intersected at two points.

\begin{tabular}{|c|c|c|c|c|c|c|c|c|c|c|c|c|c|c|c|c|c|c|c|c|}
\hline \multirow[t]{2}{*}{ Run no } & \multicolumn{2}{|c|}{$\mathrm{TI}_{1}$} & \multicolumn{2}{|c|}{$\mathrm{TI}_{2}$} & \multicolumn{2}{|c|}{$\mathrm{TI}_{3}$} & \multicolumn{2}{|c|}{$\mathrm{TI}_{4}$} & \multicolumn{2}{|c|}{$\mathrm{TI}_{5}$} & \multicolumn{2}{|c|}{$\mathrm{TI}_{6}$} & \multicolumn{2}{|c|}{$\mathrm{TI}_{7}$} & \multicolumn{2}{|c|}{$\mathrm{TI}_{8}$} & \multicolumn{2}{|c|}{ TI9 } & \multicolumn{2}{|c|}{$\mathrm{TI}_{10}$} \\
\hline & $X_{1}$ & $X_{2}$ & $X_{1}$ & $X_{2}$ & $X_{1}$ & $X_{2}$ & $X_{1}$ & $X_{2}$ & $X_{1}$ & $X_{2}$ & $X_{1}$ & $X_{2}$ & $X_{1}$ & $X_{2}$ & $X_{1}$ & $X_{2}$ & $X_{1}$ & $X_{2}$ & $X_{1}$ & $X_{2}$ \\
\hline R1 & - & - & - & - & - & - & - & - & - & - & - & - & - & - & - & - & 196 & 15 & - & - \\
\hline R2 & - & - & - & - & - & - & - & - & - & - & - & - & 31 & 5 & - & - & 62 & -57 & - & - \\
\hline R3 & - & - & - & - & - & - & - & - & - & - & - & - & - & - & - & - & - & - & - & - \\
\hline R4 & - & - & - & - & - & - & - & - & - & - & - & - & 169 & 19 & - & - & - & - & - & - \\
\hline R5 & - & - & - & - & - & - & - & - & - & - & - & - & 71 & 40 & - & - & 148 & 39 & - & - \\
\hline R6 & - & - & 98 & -8 & - & - & 58 & 32 & - & - & - & - & - & - & - & - & - & - & - & - \\
\hline R7 & - & - & 141 & -21 & - & - & 70 & -50 & - & - & - & - & 81 & 18 & - & - & - & - & - & - \\
\hline R8 & - & - & 153 & 15 & - & - & - & - & - & - & - & - & - & - & - & - & - & - & - & - \\
\hline R9 & - & - & 174 & 10 & - & - & - & - & - & - & - & - & - & - & - & - & - & - & - & - \\
\hline R10 & - & - & - & - & - & - & - & - & 160 & 40 & - & - & - & - & - & - & - & - & - & - \\
\hline R11 & - & - & - & - & - & - & - & - & - & - & - & - & 38 & 15 & - & - & - & - & - & - \\
\hline R12 & - & - & - & - & - & - & - & - & - & - & - & - & - & - & - & - & - & - & - & - \\
\hline R13 & - & - & 125 & 20 & - & - & - & - & - & - & - & - & - & - & - & - & - & - & - & - \\
\hline R14 & - & - & - & - & - & - & 138 & -35 & - & - & - & - & - & - & - & - & - & - & - & - \\
\hline R15 & - & - & - & - & - & - & - & - & - & - & - & - & - & - & - & - & - & - & - & - \\
\hline R16 & - & - & - & - & - & - & - & - & - & - & - & - & - & - & - & - & - & - & - & - \\
\hline R17 & - & - & - & - & - & - & - & - & - & - & - & - & - & - & - & - & - & - & - & - \\
\hline R18 & - & - & 205 & 16 & - & - & - & - & 130 & -96 & - & - & 96 & 22 & - & - & - & - & - & - \\
\hline R19 & 120 & -178 & - & - & - & - & 74 & -91 & - & - & - & - & 43 & 20 & - & - & - & - & - & - \\
\hline R20 & - & - & - & - & - & - & 103 & 28 & - & - & 158 & 58 & - & - & - & - & 66 & -16 & & \\
\hline R21 & - & - & - & - & - & - & - & - & 111 & -79 & - & - & - & - & - & - & - & - & - & - \\
\hline R22 & - & - & - & - & - & - & - & - & - & - & - & - & - & - & - & - & - & - & - & - \\
\hline Max & 120 & -187 & 205 & -21 & - & - & 138 & -91 & 160 & -96 & 158 & 58 & 169 & 40 & - & - & 196 & 57 & - & - \\
\hline Min & 120 & -187 & 98 & 10 & - & - & 58 & 28 & 111 & 28 & 158 & 58 & 31 & 5 & - & - & 62 & 39 & - & - \\
\hline Average & 120 & -187 & 149 & 5 & - & - & 89 & -23 & 134 & -45 & 158 & 58 & 76 & 20 & - & - & 118 & -5 & - & - \\
\hline Stdev & - & - & 37 & 16 & - & - & 32 & 53 & 25 & 74 & - & - & 48 & 10 & - & - & 65 & 41 & - & - \\
\hline Median & 120 & -187 & 147 & 13 & - & - & 74 & -35 & 130 & -79 & 158 & 58 & 71 & 19 & - & - & 107 & -1 & - & - \\
\hline
\end{tabular}

Cite this article as: Goel, A., Kumar, P., 2015. Zone of influence for particle number concentrations at signalised traffic intersections, Atmospheric Environment 123, 2538. Online Link: http://dx.doi.org/10.1016/j.atmosenv.2015.10.054 
Cite this article as: Goel, A., Kumar, P., 2015. Zone of influence for particle number concentrations at signalised traffic intersections, Atmospheric Environment 123, 25-38. 